\title{
Nonlinear Optical Properties of CdSe and CdTe Core-Shell Quantum Dots and Their Applications
}

\author{
Um e Kalsoom, Rongxing Yi, Junle Qu and Liwei Liu* \\ Key Laboratory of Optoelectronic Devices and Systems of Guangdong Province and Ministry of Education, College of Physics \\ and Optoelectronic Engineering, Shenzhen University, Shenzhen, China
}

The strong nonlinear optical behavior of low-dimensional materials, such as quantum dots and core-shell quantum dots, has been a topic of intense research in recent years. As quantum dots have tunable emission via changes in their sizes, they are potentially useful in photo-electronics, photovoltaic nonlinear optics, light-emitting diode fabrication, and laser protections. Variation among core and shell shape and size, along with the chemical composition of quantum dots, define their enhanced nonlinear optical properties. Some specific nonlinear optical properties, such as nonlinear refraction, optical limiting, saturable absorption, reverse saturable absorption of CdTe and CdSe quantum dots (QDs), as well as core-shell QDs and their applications, were assessed in this paper.

\section{OPEN ACCESS}

Edited by:

Zhong-Jian Yang,

Central South University, China

Reviewed by:

Venugopal Rao Soma, University of Hyderabad, India

Raffaella Signorini,

University of Padua, Italy

*Correspondence:

Liwei Liu

liulw@szu.edu.cn

Specialty section:

This article was submitted to

Optics and Photonics,

a section of the journal

Frontiers in Physics

Received: 30 September 2020 Accepted: 01 February 2021

Published: 20 April 2021

Citation:

Kalsoom Ue, Yi R, Qu J and Liu L (2021) Nonlinear Optical Properties of CdSe and CdTe Core-Shell Quantum

Dots and Their Applications.

Front. Phys. 9:612070.

doi: $10.3389 /$ fphy.2021.612070
Keywords: quantum dots, core-shell quantum dots (QDs), CdSe, CdTe, nonlinear optics

\section{INTRODUCTION}

Growing energy demands in the modern era have inspired great technological advancements and the concurrent ever upcoming semiconductor industry limits have made consistent developments in new functional materials, which are one of the most demanding important challenges of today's world. In the past two decades, low-dimensional materials such as QDs and core-shell QDs have been extensively studied owing to their valuable characteristics in practical applications and fundamental research. Significant progress has been made toward various QDs and core-shell QDs, particularly II-VI group compounds such as CdSe and CdTe, due to their high photoluminescence (PL), good quantum yield (QY), and optical nonlinearities via confining the electrons in smaller regions than their natural delocalization length in bulk.

Crystals with a nano-meter size have undue importance for the scientific society as these crystals offer opportunities to study different properties of materials concerning their sizes and vastly exciting polarizable states, and their unusual photochemical effects make a foundation for nonlinear optical properties $[1,2]$. Quantum dots research has been successful since these materials were first synthesized in the 1980s [3]. Quantum dots have different electronic and optical behaviors as bulk particles on account of quantum confinement. Owing to their remarkable difference in physical properties and enhancement of third-order optical nonlinearities, they have attracted considerable attention [4-7]. Detailed reviews of the electronic properties of QDs were presented by Yoffe $[8,9]$ and the optical nonlinearities of QDs were reviewed by Banfi's group [10]. Till now, numerous kinds of QDs have been studied including ZnS [11], CdSe [12], and CdTe [13]. Recently, research on quantum dots has evolved from fundamental materials science to nonlinear optical and biological applications [14-17].

Quantum dot optical properties could be tailored by changing their sizes to unlock numerous opportunities to improve photonic devices such as photovoltaic, solar cell, and photodetectors. So, it 
is conceivable to develop optical switches and other kinds of devices to manipulate light with light if fast optical nonlinearities of the QDs are to be used [18]. Meanwhile, in the mid-1990s, core-shell nanoparticles, also known as artificial atoms [19], have received considerable attention due to their valuable chemical and physical behaviors. Quantum dots' photostability and photoluminescence quantum yield can be improved via growing a wide bandgap semiconductor materials shell over the core and, due to various edge alignments of valence and conduction bands, the core/shell quantum dots were classified in different categories which are explained in the following sections [20,21]. Usage of the shell transforms QDs surface chemical [22], reactive [23], magnetic [24, 25], and catalytic properties [26]. For example, CdSe QDs coated with CdS or ZnTe and CdTe QDs coated with CdSe (type-1 core/shell QDs) have been studied for luminescent properties enrichment. $\mathrm{ZnS}$ is utilized as a shell material as it is non-toxic and has very favorable biocompatibility, while core CdTe QDs (type-II core-shell quantum dots) are more preferably considered QDs owing to their photoluminescence properties [27, 28]. CdSe QDs' emission intensity rises if CdSe (or CdTe) QDs were enclosed in a shell of materials with a large bandgap, such as ZnS, to form a CdSe/ZnS (or CdTe/ZnS) core-shell QD. The experimental results of Han Pan et al [29] display the nonlinear absorption properties of CdSe/ZnS QDs and such QDs saturable absorption depicts them as potential saturable absorbers. High-quality CdSe-based QDs are used as favorable single-electron transistors for solar, lightemitting diodes and bio-imaging applications [30]. Various NLO properties of quantum dots were depicted based on their shape and size, and such kinds of properties remain closely associated with the growth time as Li et al. found growth timedependent QDs absorption optical spectrum. During the preparation of quantum dots, the nonlinear optical properties of quantum dots are strictly associated with the growth time. For example, Li et al discovered the QDs absorption optical spectrum centered on time growth, and a longer growth time of QDs causes a progressive increase in peak values from $477 \mathrm{~nm}$ to $608 \mathrm{~nm}$. They also found fluorescence value change $(524 \mathrm{~nm}, 558 \mathrm{~nm}, 592 \mathrm{~nm}$, $612 \mathrm{~nm}$, and $667 \mathrm{~nm}$ ) when the CdTe QDs growth time changes from 1 to $28 \mathrm{~h}$. Amendment in QDs size and shape reflected the different nonlinear optical properties [28].

The present effort deals with CdSe and CdTe QDs NLO effects, providing an inclusive NLO characterization spectral range of these QDs by computing the size influence of these QDs on their NLO properties [31]. Low-dimensional materials, such as QDs and core-shell QDs, have generated considerable concerns due to their nonlinear optical properties [32]. CdSe QDs nonlinear absorption and nonlinear refraction were examined with the Z-scan technique in a wide spectral range through the femtosecond laser system. Particular studies led us to microscopic, macroscopic, and molecular weight NLO parameter values, which are fairly promising for further QDs applications utilizing both three-photon and two-photon absorption in the selected wavelength ranges, as explained in Ref. [31].

\section{BASIC NLO CHARACTERISTICS}

Nonlinear optical effects have been systematically investigated and exploited in the realization of commercial optical devices in various technological and industrial applications in the last two decades. After the invention of the ruby laser by Maiman et al, nonlinear optics was developed in the 1960s, which further discloses the nonlinear optical phenomenon in various materials. Nonlinear optics consider applied electromagnetic field interactions in several materials, which creates a novel electromagnetic field with changed frequencies, phases, and other physical quantities [33-37]. Particular nonlinear interactions lead to several optical occurrences, such as second-harmonic generation (SHG), third-harmonic generation (THG), high harmonic generation (HHG), fourwave mixing (FWM), saturable absorption (SA), and reverse saturable absorption (RSA). Nonlinear optical effects fall into two categories: parametric and non-parametric nonlinear effects. In the parametric nonlinear interactions, the material quantum state is not changed by the interaction with the optical field, and as a consequence energy and momentum are conserved in the optical field, making polarization-dependent and phase matching important. The parametric process includes second-order susceptibility, second-harmonic generation, sum-frequency generation, difference-frequency generation, and optical rectification. Third-order susceptibility involves third-harmonic generation and an intensity-dependent refractive index. On the contrary, the non-parametric nonlinear effects are accompanied by the transfer of populations to higher excited states. The nonparametric nonlinearities include two-photon absorption (2 PA) and three-photon absorption (3 PA) (third and fifth-order nonlinearities, respectively). When optical fields are not too large, particular nonlinearities can be described by the expansion of the Taylor series of the dielectric polarization density $\mathrm{P}(\mathrm{t})$ and time $(\mathrm{t})$ in terms of an electric field $\mathrm{E}(\mathrm{t})$, as shown in Eq. 1.

Once the material is affected by an applied optical field, polarization $\mathrm{P}(\mathrm{t})$ will expand as a power series in terms of optical field strength $\mathrm{E}(\mathrm{t})$ :

$$
P=\varepsilon_{0} \chi^{(1)} E+\chi^{(2)} E^{2}+\chi^{(3)} E^{3}+\cdots
$$

In Eq. 1, $\chi^{(n)}$ is the $n_{t h}$ order susceptibility (material coefficient) of a given medium. Here $\chi^{(1)}$ indicates conventional linear behavior comprising absorption plus refraction. It must be noted that $\mathrm{P}(\mathrm{t})$ and $\mathrm{E}(\mathrm{t})$ are used as scalar and $\chi^{n}$ is $(\mathrm{n}+1)$ tensor that depicts interactions that constitute a polarization-dependent nature as well as nonlinear materials symmetries. All nonlinear optical phenomena are accompanied by a change in polarization. When a material is affected by the applied electric field, the induced polarization generates an internal electric field that modifies the subsequent polarization and the applied electric field. This interrelationship is the origin of nonlinear polarization. The higher terms for $\mathbf{E}(t)$ are not linear; these terms are referred to as the nonlinear polarization and give rise to nonlinear optical effects. Also note that for small fields the polarization will be nearly equal 


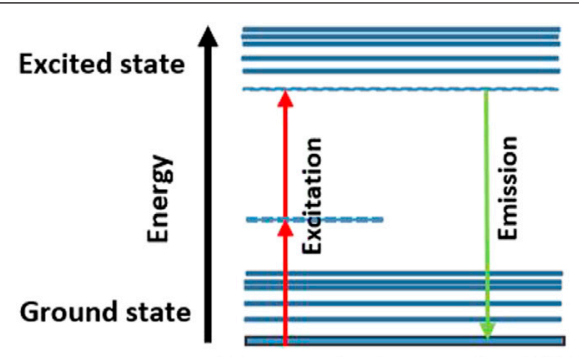

Second Harmonic Generation (SHG)

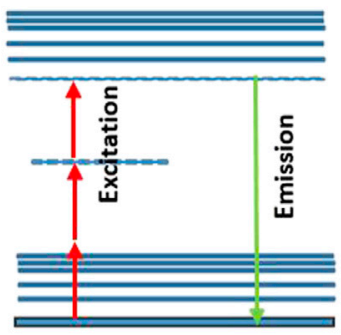

Third Harmonic Generation (THG)

FIGURE 1 | |llustration of SHG and THG process.

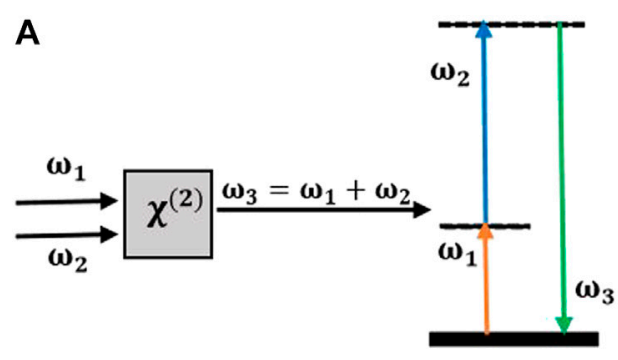

B
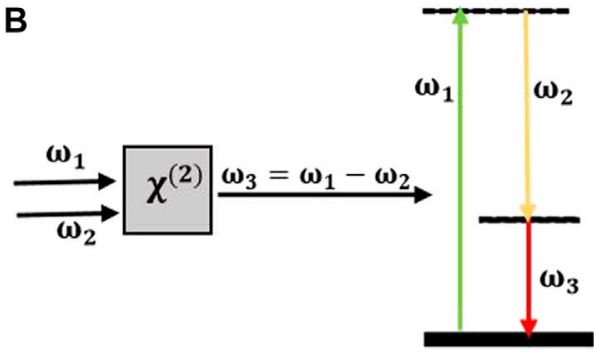

FIGURE 2 | Illustration of (A) SFG and (B) DFG process.

to a linear response while for stronger fields, nonlinear effects become more important.

Higher-order terms in Eq. 1 become noticeable when an intense optical field is used, creating frequencies of radiations dissimilar to incident light frequencies. Second-order NLO susceptibility $\chi^{(2)}$ refers to second-order NLO behavior such as Second-harmonic generation (SHG), Difference frequency generations (DFG), and Sum frequency generations (SFG). In SHG, an input wave of frequency $\omega$ produces an output at double frequency $2 \omega$, shown in Figure 1. In DFG and SFG, two input photons with two different frequencies, $\omega_{1}$ and $\omega_{2}$ can produce a new photon. A new photon of frequency $\omega_{3}=\omega_{1}+\omega_{2}$ is known as the SFG process. However, in the DFG process, the annihilation of pump photons at the frequency $\omega_{1}$ and $\omega_{2}$ gives rise to a photon of frequency $\omega_{3}=\omega_{1}-\omega_{2}$, shown in Figure 2. Third-order NLO effects include third-harmonic generation (THG) (Figure 1), four-wave mixing (FWM), and saturable absorption (SA) phenomenon, which arises due to third-order susceptibility. The $n_{t h}$ order susceptibility $\chi^{(n)}(\mathrm{n}<5)$ describes the high-order multiphoton scattering/ luminescence/absorption, and high harmonic generation (HHG) phenomenon. For higher-order terms, the nonlinear effect becomes weaker as higher-order represents the involvement of more photons. High-order nonlinear effects can be observed at high irradiance, since usually $\chi^{(2)}>\chi^{(3)}>\chi^{(4)}>\chi^{(5)}$ and so on. In the case of third-order nonlinear effects, an increase in " $n$ " causes a decrease in the strength of nonlinear interactions so, due to this, the thirdorder nonlinear effects are the most commonly utilized processes for various applications such as frequency conversion, imaging, modulators, and ultrafast lasers [38].

Multiphoton absorption is a nonlinear phenomenon in which simultaneous absorption of two or more photons is observed in a single event. The most common multiphoton absorption processes are the degenerate two- and three-photon absorption process. Multi-photon absorption-based techniques have been developed to attain several photonic applications such as biological imaging, data storage, and optical power limiting. Three-photon absorption (3 PA) has several advantages such as higher spatial resolution, contrast image, high penetration ability, and reduction of scattering losses when dealing with longer wavelengths. Higher-order nonlinear optical effects have prior properties as compared to the single-photon absorption (depend upon first order susceptibility) and twophoton absorption (Third-order susceptibility) [39]. Twophoton absorption is a third-order nonlinear process that depends upon the intensity of light and can dominate over linear absorption at high intensities.

Various nonlinear optical phenomena can be applied for 3D imaging of semiconductors in which two-photon absorption charge carriers can be generated spatially confined in semiconductor devices. Another area of research for nonlinear optical phenomenon is optical power limiting in which a material that exhibits a strong nonlinear effect absorbs more light for higher intensities, such as that above a certain limit of input intensity the output intensity achieves a constant value so particular materials can be used to control noise in laser beams and also protect sensitive equipment, such as sensors, because the nonlinear absorption 

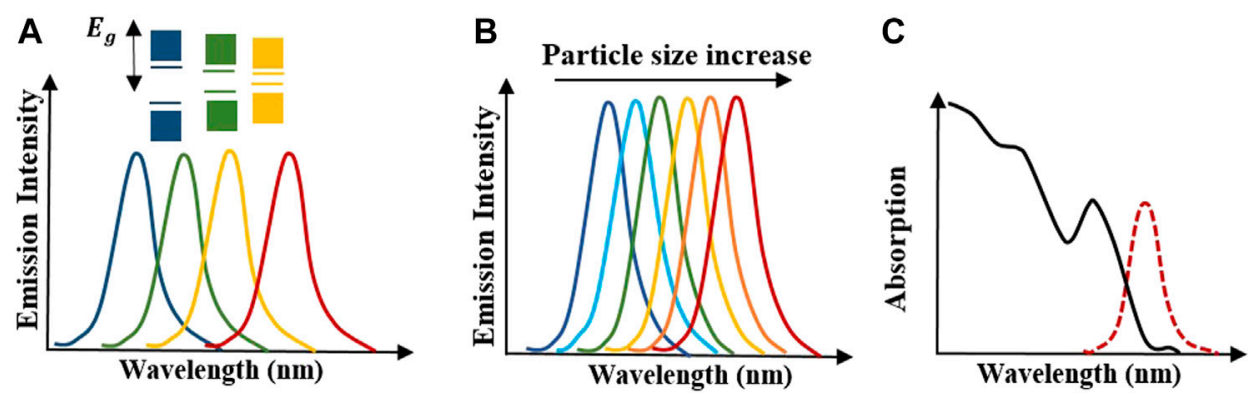

FIGURE 3 | Quantum dot optical properties (A, B) Bandgap energy dependency on particle size. (C) Their emission multispectral ability [47].

coefficient increases with the increase in input power. The increase of sample temperature due to absorption results in a nonlinear absorption coefficient and optical limiting effect. To behave as an active optical limiter, the nonlinear optical medium must possess high linear transmittance, low limiting threshold, fast response time, broadband response, and low optical scattering. Nonlinear optical properties also play an important role in the progress of optical limiting devices. Optical limiting has different origins: RSA which takes place due to enhanced absorption is responsible for optical limiting in metal compounds and fullerenes and $2 \mathrm{PA}$ is responsible for optical limiting in semiconductor structures. One of the main sources of optical nonlinearities is the excited-state-induced process, which has potential application for optical limiting; study of these states in terms of enhanced populations of various optical nonlinear solutions is worth continuing by using variation among sizes of their nonlinear optical responses at off-resonant conditions [40]. Optical limiting behavior can be enhanced by choosing the appropriate parameters, such as detuning and the tunneling rate, from which the system will reveal good optical limiting properties in certain intensity regions which can be attributed to an RSA mechanism. The interdot tunneling can affect the system's optical limiting performance and the threshold of the limiting behavior can be controlled by increasing the tunneling coupling; indeed, the threshold would be a function of input voltage allowing optimization of the optical limiting behavior. Particular controllable limiting behavior, associated with the high transmittance at low intensities and low threshold optical limiting behavior, makes quantum dots materials promising for optical limiting development [41].

\section{PHYSICAL PROPERTIES AND CHARACTERISTICS OF QUANTUM DOTS (QDs)}

Quantum dots (QDs) are smart materials that consume smaller radii than bulk exciton Bohr radius and their properties are transitional between bulk matter and molecules [42-44]. Consequently, QDs are recognized as the ideal basic physical material among single atom and ordinary materials [45] and they exhibit massively different electronic properties owing to their small sizes. Commonly, QDs are composed of IV, II-IV, III-V, or IV-VI group elements and their size can be precisely controlled via monitoring temperature, reaction time, and ligands. Quantum dots are usually comprised of a different number of atoms that commonly originate at the surface of nano-crystals dependent upon their sizes [46]. Quantum dots of smaller sizes depict shorter wavelengths with enormous bandgap energy for high energy levels. The emitted light is proportional to the kinetic energy and energy bandgap, as shown in Figure 3B [47].

Quantum dots bandgap energy is correlated with their size, so when the dimension of the particle decreases, the band energy increases, and the illustration of particular dependence on QDs size is shown in Figure 4. When quantum dots size is reduced, some important properties, such as their optical properties, are drastically altered, as reduction of particle size causes a split in the band energy distribution and, therefore, higher bandgap energy is obtained. As the size of quantum dots decreases the energy spectrum will change from a continuous to a discrete spectrum. For smaller particles, as the bandgap increases (which physically means that a large amount of energy is required to separate the electron and hole to activate the material) a large amount of energy is released at shorter wavelengths when the electron and hole combine. For larger quantum dots the phenomenon is reversed, as larger quantum dots constitute a smaller bandgap or energy gap so less energy is required to activate the material through electron-hole separation. For the deactivation of the material, the electron and hole combine and releases a small amount of energy at larger wavelengths. This shows that, by controlling the particle dimensions, one can achieve different band structures that represent that QDs have size-tunable optical properties. Recently, the electronic structures that originated from interfacial effects along with quantum confinement have been massively investigated as they exhibit a fast response time and large optical nonlinearities [48, 49].

\section{CHARACTERISTICS OF CORE-SHELL QUANTUM DOTS}

Quantum dots have a diameter of few nanometers, so they exhibit a very high surface-to-volume ratio (about $80 \%$ of the atoms reside on the surface). In quantum dots, a high surface-to-volume ratio (originated from imperfections and dangling bonds) results in the band structures that in turn can be utilized for the 

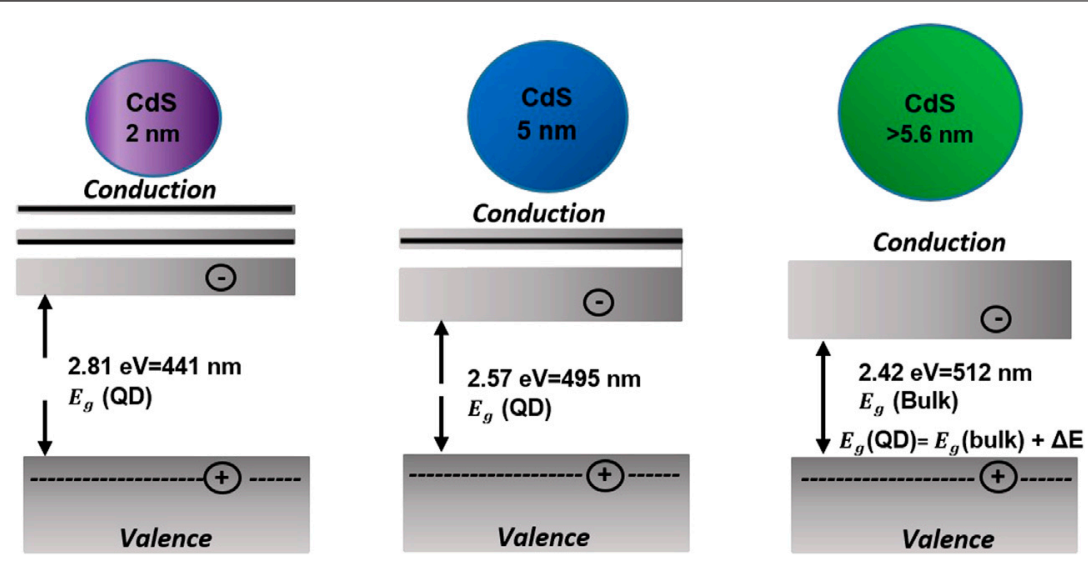

FIGURE 4 | Diagram illustration of the solid band structures of QD size variation effect on the transition energies. CdS QDs of different sizes, such as >5.6, 5, and $2 \mathrm{~nm}$, exhibit bandgap energies of 2.42, 2.57, and $2.81 \mathrm{eV}$, respectively. A decrease in the size of CdS QD caused an increase in bandgap energy anda split in the band energy distribution [48].
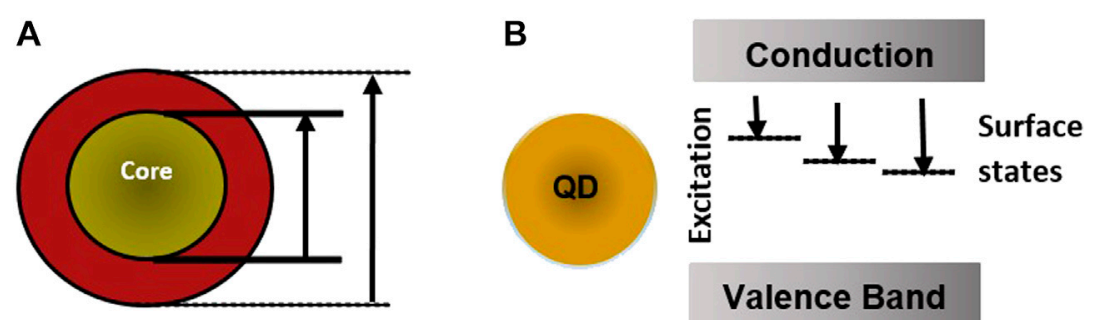

FIGURE 5 | Diagram illustration of the solid band structures: (A) Within the QDs bandgap, the surface traps their electronic transitions. (B) Core-shell QD electronic structure made of two semiconductors forming heterojunctions [48].

comparisons of two different quantum dot systems. These unsaturated dangling bonds act as efficient charge traps that drastically decrease the quantum yield [50]. The high surface-tovolume ratio of quantum dots is originated from imperfections and dangling bonds make particular band structures that are a basic approximation of definite QDs systems. Electronic energy states of such surface trap sites are localized in QDs bandgap, as depicted in Figure 5B. An abundance of surface states causes instability. To remove this particular instability, two or more semiconductor combinations forming hetero-junctions have been utilized with a core surrounded by a wider bandgap semiconductor shell which passivates QDs surface states, as shown in Figure 5A [48]. Structurally, core-shell QDs were prepared from the core (II-VI or III-V group atoms especially CdTe and CdSe) coated with semiconductor shells (usually $\mathrm{ZnS}$ ) to develop optical properties. The outermost part of particular core-shell QDs is composed of ligand molecules, which are an utmost acute parameter in determining the morphology, size, solubility, stability, and distribution of QDs particles.

The core is the portion of quantum dots that represents optical properties besides semiconducting behavior. To enhance quantum yield and optical properties of QDs, inorganic shells can be utilized to neutralize surface free atoms and create a physical barrier among the core and external factors that form

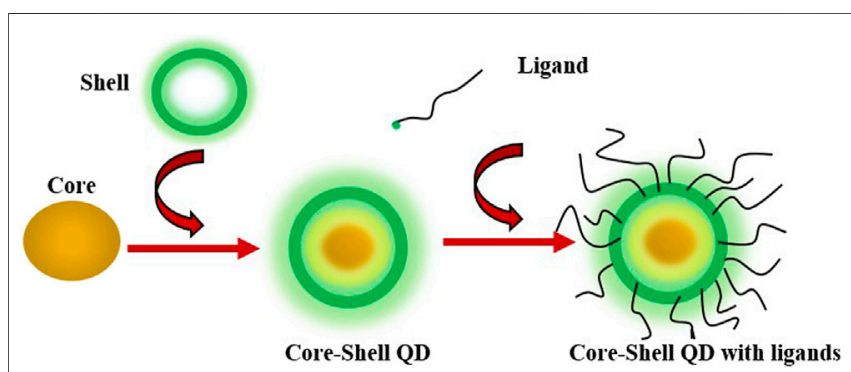

FIGURE 6 | Core-shell QDs interaction functionalized with ligand molecule [54].

surface defects [51-54]. As shown in Figure 6, the coating of the shell layer above the core QDs to increase quantum yield (QY) and stability has been confirmed by Hines and Guyot-Sionnest. $\mathrm{CdSe} / \mathrm{ZnS}$ QDs QY is $40 \%$ at room temperature [20, 55]. Rosenthal et al. carried out widespread work and discovered that shell materials are not constantly deposited around the core $[56,57]$.

A specific choice of core and shell material depicts several electronic configurations dependent upon how valence and conduction band edges line up in the shell to the core. This is 


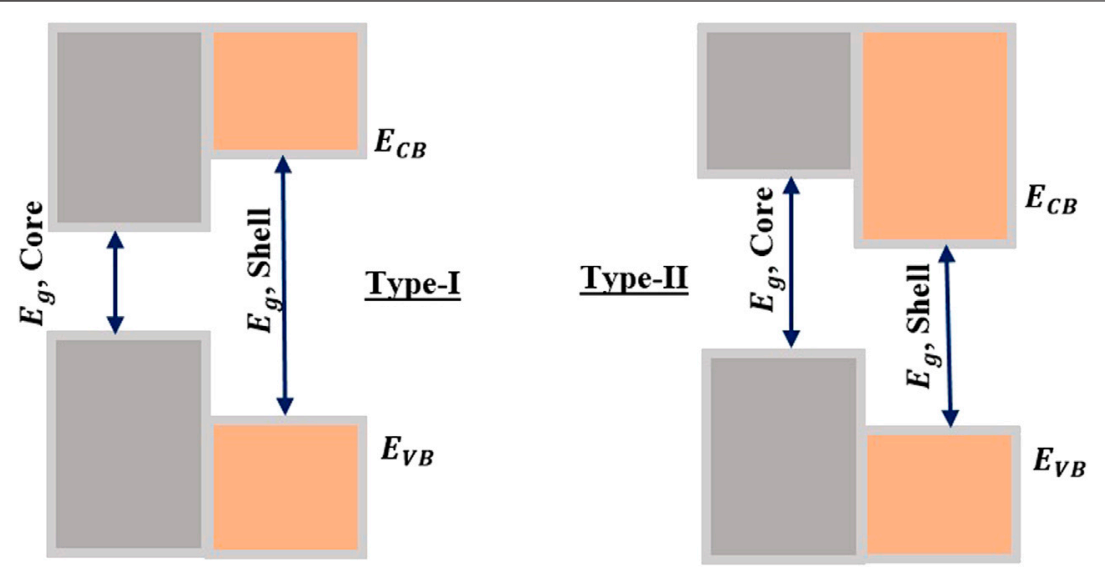

FIGURE 7 | Representation of the Type-I and Type-II core-shell quantum dots based on the band alignment [77].

TABLE 1 | Physical properties of Type-I and Type-II core-shell quantum dots based on band alignment [77].

\begin{tabular}{|c|c|c|}
\hline Parameters & Type-I & Type-II \\
\hline Bandgap & $\begin{array}{l}\text { The bandgap of the shell is greater as compared to the core, while the } \\
\text { bandgap of the core falls within the bandgap of the shell }\end{array}$ & $\begin{array}{l}\text { The conduction band edge of the shell is within the bandgap of the core } \\
\text { and the valence band edge of the core is within the bandgap of the shell }\end{array}$ \\
\hline $\begin{array}{l}\text { Excited electron/holes } \\
\text { positions }\end{array}$ & Excited electrons and holes are completely confined in the core region & $\begin{array}{l}\text { One charge carrier (either an excited hole or electron) is confined to the } \\
\text { core while the other is confined to the shell }\end{array}$ \\
\hline Quantum yield (QY) & Higher QY & Lower QY \\
\hline Stability & Long term stability & Poor stability \\
\hline $\begin{array}{l}\text { Average absorption } \\
\text { range }\end{array}$ & $400-500 \mathrm{~nm}$ & $600-800 \mathrm{~nm}$ \\
\hline $\begin{array}{l}\text { Average emission } \\
\text { range }\end{array}$ & $430-600 \mathrm{~nm}$ & $700-1000 \mathrm{~nm}$ \\
\hline Limitations & $\begin{array}{l}\text { Reduction in fluorescence QY is observed due to the trapping of charge } \\
\text { carriers by the shell }\end{array}$ & One of the excited electrons or holes leaks to the surface \\
\hline Construction/Materials & $\mathrm{CdSe} / \mathrm{ZnS}, \mathrm{CdSe} / \mathrm{CdS}, \mathrm{CdS} / \mathrm{ZnS}$ & $\mathrm{CdTe} / \mathrm{CdSe}, \mathrm{CdSe} / \mathrm{ZnTe}, \mathrm{CdSe} / \mathrm{ZnSe}$ \\
\hline
\end{tabular}

very important for developing efficient nonlinear optical applications [50]. Quantum dots with different NLO effects could be detected owing to the core materials' arrangement. QDs, especially CdTe and CdSe, nonlinear optical properties have also been observed (mainly third-order optical nonlinearity) [58-61] as particular QDs optical properties, such as size-tunable emission spectra, high photostability, bright fluorescence, and broad-range ultraviolet excitation, make them suitable candidates for different applications such as imaging tools, diagnostics, photobleaching, and chemical retardation [62, 63]. Nonlinear optical properties of $\mathrm{CdTe} / \mathrm{CdS}$ core-shell QDs have been investigated in the work of E. Saievar-Iranizad et al. [64]. In a particular study, the positive nonlinear refractive coefficient was obtained that displays a self-focusing effect in the sample, and a negative sign was obtained for the nonlinear absorption coefficient. D. Chauvat et al. [65] revealed coherent SHG from single core-shell CdTe/CdS QDs with a diameter of 10-15 nm. The intrinsic coherence and orientation sensitivity of the SHG process of particularly small size nonlinear active core-shell QDs are well adapted for optical near-fields ultrafast probing with high resolution as well as for orientation tracking for bioimaging applications. Desirable features of core-shell QDs, such as photostability, excellent color purity, and tunable photoluminescence (PL), make them appropriate for use in electronics [66, 67], lasers [68, 69], quantum dots lightemitting diodes (QLEDs) [70-72], medical sciences [73, 74], bioimaging, and bio labeling $[71,75]$.

Selected core-shell materials' conduction band (CB) and valence band $(\mathrm{VB})$ edges, along with the nature of the bandgap, defines core-shell QDs systems [76]. According to the valence and conduction bands of core and shell structures in core-shell quantum dots, they were divided into further categories. Type-I and Type-II core-shell QDs usually comprise various physical properties and different semiconductor combinations, as shown in Figure 7; Table 1. Type- 1 is more favorable as compared to type-II because of their better stability, higher quantum yield, and no leakage of excited electrons. Subsequently, to develop optoelectronic properties, shells of various compositions could be utilized for passivation of an active surface of the core to diminish surface defect states and improve PL, QY, and NLO properties observed to be enhanced [77]. 


\section{STRONG CONFINEMENT EFFECTS IN QUANTUM DOTS AND CORE-SHELL QUANTUM DOTS}

In the bulk structure of semiconductors, charge carriers (electrons and holes) have a continuous distribution of energy states and their motion is not confined. However, in quantum well (QW) structures, these charge carriers are confined and their energy states are quantized in one dimension. Further confinement and quantization of energy in two dimensions is achieved in quantum structures where the motion of charge carriers is confined and energy states are quantized in two dimensions. Confinement of electrons in three dimensions and hence quantization of its energy states can be established in quantum dot structures [78]. However, as opposed to the bulk material, the size of the quantum dots greatly impacts the electronic and optical properties. This is particularly important for semiconductor QDs where the Fermi level lies between two bands and therefore the band edges are key to the electronic and optical properties.

As the band structure in materials develops fully only in the bulk limit, particle size strongly influences the bands and the bandgap and, therefore, the optical and electronic properties. So, one of the most important phenomena at the nanoscale is the quantum size effect. This effect arises when the physical dimensions of nanocrystals become smaller than the characteristics length of the charge carriers (electron and hole) quantum states in the ground or their excited states. The coulomb attraction among appositively charge carriers derives spatial localization of bound electrons-hole pairs. These effects make the optical emission and absorption properties of quantum dots size-dependent, with band peaks shifting to shorter wavelengths at smaller sizes [50]. Photon emission of semiconductor quantum dots can be generally described as a transition across their electronic bandgap. Quantum dots have a tunable bandgap due to the concept known as quantum confinement. Quantum confinement affects the absorption and emission of photons from the dot. Thus, the absorption edge of a material can be tuned by control of the particle size; as size is reduced to the nanoscale range, the exciton binding energy increases because of the electron and hole increased spatial overlap (quantum confinement) When QDs bulk exciton Bohr radius and nanosize were of equal magnitude, the movement of the hole or electron is restricted in three-dimension leading to energy level separations. Such electronic structure imbues QDs with superficial quantum confinement effects and nonlinear effects.

$$
a_{E B R}=\varepsilon_{r}\left(\frac{m}{\mu}\right) a_{B}
$$

In Eq. 2 " $\mu$ " means reduced mass, " $m$ " represents mass, " $\varepsilon_{r}$ " shows dielectric constant (size dependent), and $a_{B}=0.53 \times 10^{-10}$ is the Bohr radius [79]. Quantum confinement effects of the quantum dot can be categorized into two types via utilizing Eq. 2: weak confinement regime and strong confinement regime [80]. In the case of a weak confinement regime, when the QDs radius (R) is greater than the Bohr radius $\left(a_{B}\right)\left(R \gg a_{B}\right)$, for nonlinearity weakly correlated exciton are responsible. In the case of strong confinement regime $\left(R \ll a_{B}\right)$, the photoexciton electron and hole are confined. Schmitt-Rink et al. illustrates that in a strong regime state-filling effect is responsible for optical nonlinearity such as when the diameter of quantum dot is smaller in size than exciton, large optical nonlinearities will be observed [81]. Owing to the quantum confinement effect, the ability to control QDs size predicts the controlling of QDs various properties such as absorption and emission wavelengths. For example, CdSe QDs emission wavelength can be controlled from deep red to blue via reducing the dot radius from $20 \mathrm{~nm}$ to $0.9 \mathrm{~nm}$ [82]. This implies that in strong confinement regime limits, QDs have a great potential for massively enhanced optical properties and motivated the QDs study in a particular limit. The work by V. I. Klimov et al. clearly explains that, via changing the size of quantum dot, nonlinear optical properties can be enhanced. In this work, lasing in quantum dots produces high optical gain at wavelengths of the emitting transition for close-packed solids of these dots. At wavelengths that are tunable with the size of quantum dots, narrow stimulated emission with pronounced gain threshold was observed, as expected from quantum confinement effects [83].

It is difficult to understand the interactions among excitons because QDs states are often degenerate or nearly so. For example, in lead-salt QDs, a 64-dimensional exciton manifold is present and at the band edge, minor deviations from the symmetry and band anisotropy give rise to a splitting of degenerate energy levels, especially for smaller QDs. Interband coupling and exchange interactions can further split the excitonic energy levels. Linear absorption spectrum fails to explain these interactions due to broadening caused as a result of QDs finitesize distribution which hides the signals. So to study absorption spectrum, 2D photon spectroscopy is used as it achieves high spectral resolution [84].

Quantum dot properties, such as strong light absorption properties, make them valuable for several applications such as solar cells and light-emitting devices. The features, including the One- and two-photon absorption phenomenon, are the direct consequence of the quantum confinement effect [50]. A common approach for supporting QD quantum confinement includes encapsulating QDs inside organic surfactant that indicates fluorescent quantum yield decrease. A particular yield could be overwhelmed through increasing inorganic material epitaxial layers over quantum dots core material to attain core-shell QDs that aid PL efficiency enhancement of the QDs [85]. Due to large quantum confinement in the core-shell, quantum dots result in large energy bandgap difference, so as a result PL efficiency also increases. By increasing the thickness of the shell in core-shell QDs, the nonlinear absorption coefficient enhanced drastically; similar cases have also been achieved when an impurity was replaced from the core center to shell center in core-shell QDs.

In the case of weak confinement, the exciton binding energy is much higher than the individual confinement energy of an electron or hole. The nonlinear optical properties were enhanced by large oscillator strengths, which caused the confinement. The oscillator strength increases as the size of the nanocrystal increases (as long as the excited state remains coherent). However, exciton in bulk materials almost acts as a 
harmonic oscillator that does not show any nonlinear effect. With a decrease in size, the highest nonlinearity could be achieved. The study by Nideep et al. confirmed the quantum confinement effect of CdTe quantum dots via UV-vis absorption and fluorescence spectroscopy that shows that CdTe quantum dots exhibits quantum confinement. Third-order nonlinearity was analyzed via utilizing Z-scan measurement that indicates that quantum dots possess two-photon absorption properties. These quantum dots possess good NLO properties based on which quantum dots can be used as a potential candidate for optical limiting application [86]. $\mathrm{Y}$. Hu et al. investigated the quantum confinement effect in $\mathrm{CdSe}$ microcrystals in a glass matrix in the nanosecond time domain. This study reveals evidence for electron-hole quantization in semiconductor microstructures. As a result of quantum confinement, distinct discrete absorption lines were obtained in the spectrum as microsatellite size was decreased [87].

In terms of a nonlinear optical property, the influence of quantum effects on QDs is mainly represented on behalf of increasing the polarizability and absorbance index that provides QDs with their large nonlinear optical parameters as compared to ordinary materials. As mentioned above, high order nonlinear effects depend upon the nonlinear polarization. The bandgap in quantum dots which determine the emitted light frequency range is inversely proportional to its size. When the size of quantum dots decreases, the energy levels split up or bandgap changes (as a result of quantum confinement) and, as a result, in smaller band gaps the sum of energy levels in the strong confinement regime is larger than the energy levels in the bandgaps in the weak confinement regime. As mentioned above, high order nonlinear effects depend upon the nonlinear polarization. Form Eq. $\mathbf{1}$ it is clear that nonlinear polarization depends directly upon electric field strength and when the electric field strength becomes stronger the polarization becomes stronger; as a result, the order of nonlinear susceptibilities also increases which depicts that nonlinear effects become prominent in this region of higher susceptibilities. Electric field strength is location-dependent, so as the radius of quantum dots decreases electric field strength increases and, as a result, nonlinear polarization components become stronger. This shows that nonlinear optical properties will also increase. When the radius of quantum dots increases the degree of polarization, due to nonlinearity of the light intensity measurement at a large distance from the entry point, becomes too noisy and becomes weak. So, it can be estimated that the nonlinear polarization magnitude decreases as distance increases. Core-shell quantum dots with a large quantum confinement in core-shell quantum dots result in a large energy bandgap difference, so as a result a strong electric field is required which in return will provide good nonlinear polarization effects and, as a result, nonlinear optical properties will also increase.

\section{NONLINEAR OPTICAL CHARACTERISTICS OF QUANTUM DOTS (QDs) AND CORE-SHELL QUANTUM DOTS}

The rapid development in nanotechnology and nanoscience has motivated researchers to introduce new nanoparticles that comprise enhanced nonlinear optical properties. In this case, the main goals that are ahead are: 1) To identify specific nanoparticles that exhibit nonlinear optical phenomena mentioned in Basic NLO Characteristics section; 2) understand the relationship between their structural properties and nonlinear optical properties; and 3) demonstrate how, by using these nanoparticles, the nonlinear optical properties can be enhanced or how nonlinear optical properties could be enhanced by changing the properties of nanoparticles. Nonlinear optical devices operating as switches, routers, or frequency converters are technically feasible, but they are still limited by existing nonlinear materials. At present, there is considerable research activity in the field of nonlinear optical materials. In recent years, the size distribution changes of QDs and core-shell QDs result in a change in their nonlinear optical properties, which was reported recently by $S$. Mathew et al. [88]

Similar to bulk materials, QDs exhibit non-linear optical properties such as multiphoton absorption or emission, harmonic generation, or up- or down-conversion. The most commonly observed nonlinear effect in QDs is absorption saturation and transient bleach shift at high intensities [89-92]. These non-linear optical properties have been considered potentially useful for optical limiting and switching applications [93]. Another non-linear optical phenomenon is harmonic generation, mostly based on the third-order nonlinear optical properties of semiconductor nanoparticles $[94,95]$. The third-order nonlinearity is also responsible for phenomena such as the Kerr effect and degenerate four-wave mixing (DFWM) [96]. Only a few studies have been carried out on second-order nonlinear optical properties since it is usually believed that the centrosymmetric or near centrosymmetric of the spherical nanoparticles reduces their first-order hyperpolarizability ( $\beta$ ) to zero or near zero. Using hyper-Rayleigh scattering, the second-harmonic generation in CdSe QDs has been observed [97]. Nonlinear three-photon absorption phenomenon in semiconductor quantum dots and nanocrystals has attracted major attention for various applications, such as bio labeling and imaging agents, due to the possibility of utilizing longer wavelengths for deeper penetration depths for super-resolution imaging. Three-photon absorption in semiconductors can be utilized as a limiting factor in all-optical switching applications below half bandgap. Therefore, by having accurate experimental verification of three-photon absorption in semiconductors is of great value for characterization and designing of several NLO devices. The spin-orbit coupling and intersubband transitions in quantum dots are present which may significantly alter the 3 PA process via quantum interference between different bands. To predict the spectral dependence of $3 \mathrm{PA}$ of various semiconductor quantum dots it is important to account for quantum interference between different pathways and to determine the spectral shape spin-orbit coupling [98].

As discussed earlier, the optical properties of isolated QDs can be very different from those of assembled QDs. Theoretical calculations on nonlinear optical properties of QDs superlattice solids have shown that an ideal resonant state for a nonlinear optical process is the one that has a large volume and narrow linewidth [99-101]. The calculations also showed that 
nonlinear optical responses could be enhanced greatly with a decrease in inter-particle separation distance. For quantum dots with confinement in three dimensions, luminescence upconversion has only recently been reported for CdSe [102]. Surface states have been proposed to play an important role in the up-conversion in QDs such as CdSe. The optical properties of nanoparticles are tunable throughout the visible and nearinfrared regions as a function of nanoparticle shape, surface state, and size. It is well known that the QDs optical absorption and emission properties depend upon their chemical compositions, their dimensions, and shape, so the composition of surfaces and defect distribution are of great importance [50]. At the nanoscale level, the behaviors of defects are quite important as compared to that of bulk materials and could lead to intriguing applications. Despite the presence of defects that are growth-induced in nanostructures, electrons and ion beams can be used to create ion tracks and other radiation-induced defects in them. These defects can significantly alter the nanomaterial's electronic structures and play an important role in finding out the linear as well as nonlinear optical properties at the nanoscale. In nanostructures, a high surface state modifies the chemical and physical properties, which can reflect in NLO measurements. R. Podila et al. explored the effects of defects on the nonlinear absorption behavior of carbon and $\mathrm{ZnO}$ nanostructures. This study shows that tailoring the electronic structure of carbon and $\mathrm{ZnO}$ via using defects engineering widens the scope in NLO applications. As the number of surface states increases in particular nanostructures, it causes the enhancement of nonlinear optical properties. For example, the optical limiting response is observed in CVD-grown graphene as the number of surface states will be increased. So, varying the number of surface defects will help the nanostructures to achieve nonlinear optical properties that can be utilized to achieve efficient control over the designing and fabrication of new devices for optoelectronic applications [103].

In the case of core-shell, QDs as a result of lattice mismatch between core and shell will experience coherence strain that will cause dislocation sites and surface defects/surface states at the interface of the core-shell. These defect sites then act as trap states for photogenerated charge carriers and cause a decrease in the photoluminescence quantum yield of the quantum dots. So, to increase the photoluminescence quantum yield, core and shell materials should have fewer lattice mismatches. This will reduce the surface trap states and, as a result, the photoluminescence quantum yield of core-shell quantum dots will increase.

In semiconductor quantum dots, the increment in the Coulomb binding energy was observed as confined carriers substantially enhance the overlap among electrons and holes. Therefore, when discussing the optical properties of nanostructures it is also important to consider the excitonic effects. The quantum size effect plays a substantial role in understanding the magnitude of nonlinear coefficients so in return quantum confinement also plays an important role in it. When a quantum dot radius decreases the nonlinear absorption coefficient will increase as a result of strong quantum confinement. The physical reason is that the dipole matrix increases with the reduction of quantum dot size. Another important reason is that the resonance peak shifts toward the higher energies as the size of the quantum dot decreases. It is worth mentioning that between the ground and the first excited state the energy difference increases as the size of the quantum dot decreases.

For QDs, Raman scattering can be used to study vibrational or phonon modes, electron-phonon coupling, and symmetries of excited electronic states. Raman spectra of nanoparticles have been studied in several cases, including CdSe [104]. In order to observe large third-order susceptibility in QDs, B. Zhu reported a study of the nonlinear response of CdSe QDs [105]. Different sizes of CdSe QDs have been used to examine refraction and third-order susceptibility via the Z-scan technique. Experimental results depict positive nonlinear refraction and absorption and, in the case of resonance nonlinear absorption, substantial enrichment has been observed in third-order nonlinear susceptibility. This specifies that CdSe QDs are encouraging materials for optical devices and confirm noticeable applications in nonlinear optics (NLO) and other areas as well. Studies by H. Naderi et al. [106] depict third-order nonlinear optical properties of CdTe QDs by using the singlebeam Z-scan technique. According to particular studies' data obtained from closed-aperture $\mathrm{z}$-scan, $n_{2}$ has a negative sign that shows that particular QDs exhibits self-defocusing optical nonlinearity while results of open-aperture $z$-scan show a positive sign of $\beta$ that displays the two-photon absorption phenomenon is dominant in the intensities used for particular studies. The studies of Ref. [86] depict via the Z-scan technique that CdTe QDs samples with various sizes exhibit two-photon absorption and hence are potential candidates of optical limiting properties. The goal is to find and develop materials presenting large nonlinearities and simultaneously satisfying various technological and economical requirements. NLO materials progressively show a substantial part in various photonics characteristics i.e. imaging, detection, transmission, and photon generation [96, 107].

\section{NONLINEAR OPTICAL PROPERTIES ASSOCIATED WITH INTERSUBBAND TRANSITIONS IN CORE-SHELL QUANTUM DOTS}

In semiconductor quantum dots structures, the electron motion is quantized in all three dimensions leading to discrete energy levels. A large number of optical nonlinearities were associated with intersubband transitions that occur in quantum dots as compared to the bulk materials. Studies of the third-order nonlinear susceptibilities that are associated with these intersubband transitions in $\mathrm{CdSe} / \mathrm{ZnS}$ core-shell quantum dots were displayed in this section. Consider a system of an electron confined in CdSe/ZnS core-shell quantum dot having an inner radius $R_{1}$ and outer radius $R_{2}$, as shown in Figure 8. The results obtained from these studies were shown in Figure 8, where $\left|\chi^{(3)}(-\omega ; 0,0, \omega)\right|, \operatorname{Re} \chi^{(3)}(-\omega ; 0,0, \omega)$ and $\operatorname{Im} \chi^{(3)}(-\omega ; 0,0, \omega)$ were presented as a function of photon energy $\hbar \omega$ for different 


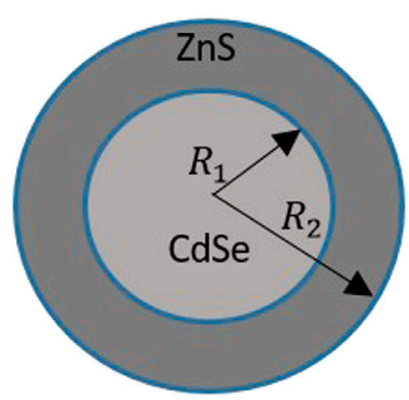

B

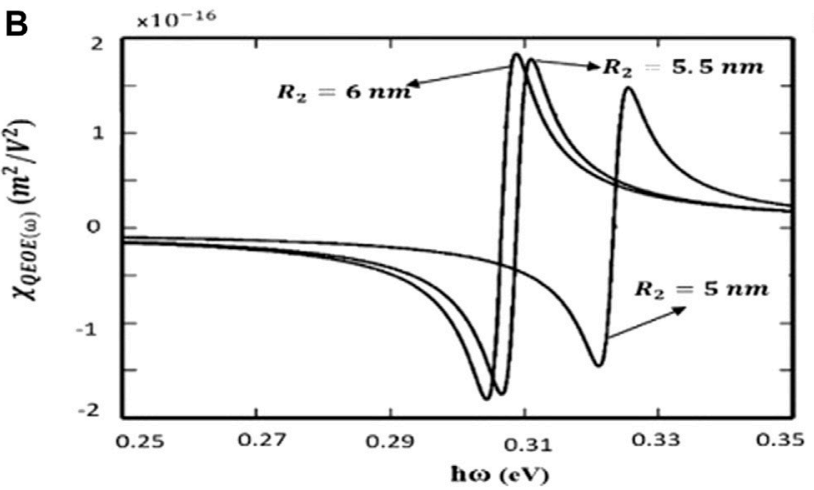

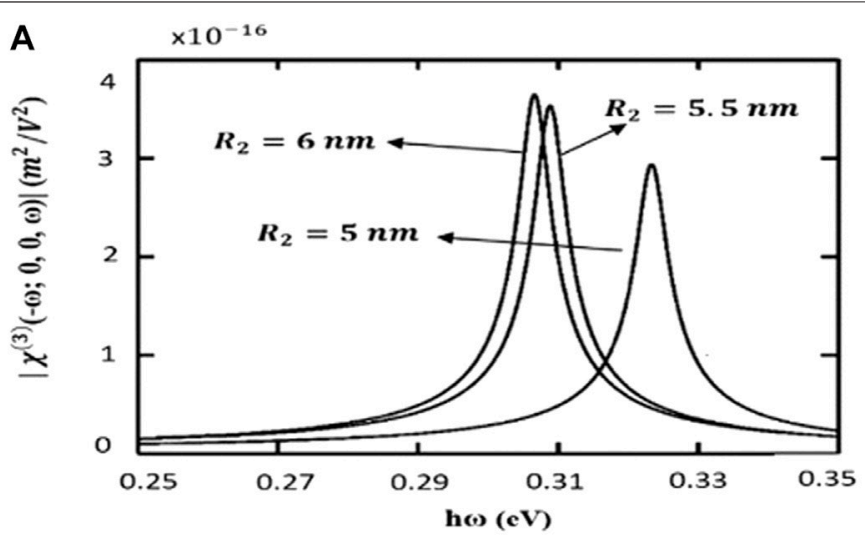

C

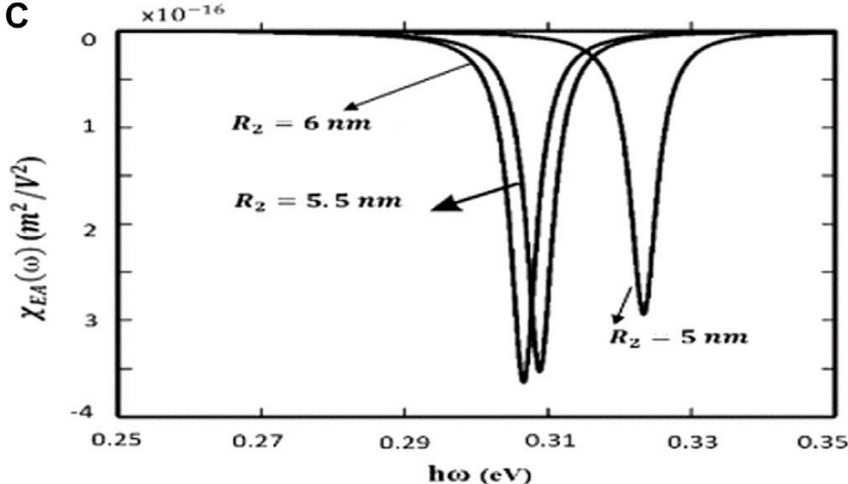

FIGURE 8 | Core-shell quantum dot pictorial diagram representing that the core is made up of CdSe quantum dot of radius $R_{1}$ and the shell is made up of $Z \mathrm{nS}$ having a radius $R_{2}$. Third-order nonlinearity of CdSe/ZnS core-shell quantum dots with different $R_{2}$ and fixed $R_{1}=4.5 \mathrm{~nm}$ were displayed above. The modulus of (A) real part (B) imaginary part $\mathbf{( C )} \chi^{(3)}(-\omega ; 0,0, \omega)$ versus pump photon are represented in the above figures [108].

outer radii with fixed inner radii and resonant $\chi^{(3)}(-\omega ; 0,0, \omega)$ magnitudes $10^{-17}-10^{-16} \mathrm{~m}^{2} / V^{2} \cdot \chi_{Q D O E}^{(3)}(\omega)=\operatorname{Re} \chi^{(3)}(-\omega ; 0,0, \omega)$ represent direct current Kerr effect and the electron absorption process. Figure 8A shows nonlinear optical susceptibilities increases and peak shifts toward smaller energies and at large $\mathrm{R}$, the peak shift is smaller while for smaller $\mathrm{R}$ the peak shift is more.

These results depict that, as a result of the quantum size effect, when $\mathrm{R}$ increases the energy distance between electronic states the conduction band becomes smaller. At larger values of $\mathrm{R}, \mu$ dipole matrix also becomes stronger. In Figure $\mathbf{8 B}, \mathbf{C}$, the most interesting feature is observed that near the resonating frequency, $\chi_{O E O E}^{(3)}(\omega)$ change its sign from $-2 \times 10^{-16} \mathrm{~m}^{2} / V^{2}$ to $+2 \times 10^{-16} \mathrm{~m}^{2} / V^{2}$ while $\chi_{E A}^{(3)}(\omega)$ always keeps negative. Results show that the third-order nonlinear susceptibilities greatly depend upon the size of the core radius. Quantum dots exhibit large optical nonlinearities due to the quantum size effect and, as a result, resonant peak shifts to lower frequencies. So, via changing the radii of core in core-shell materials nonlinear optical properties can be enhanced just because of electron confinement in the core and because there is nearly no electron in the shell barrier so as a result the electronic wave function probability distribution mainly concentrates in a core part of the core-shell quantum dots. Based on these results, quantum dots nonlinear optical properties will greatly impact the optical devices [108].

\section{CdSe AND CdTe CORE-SHELL QUANTUM DOTS NONLINEAR OPTICAL PROPERTIES AND APPLICATIONS}

Quantum dots, due to their nanoscale size, are promising 0-D materials. Quantum dot specific properties allow them to act in numerous applications as NLO and electro-optical devices. The quantum confinement effect becomes substantial to surface modification and manipulation of quantum dots diameter that allows for the control of various properties of the dot. Nanoscale QDs show an important role in the reduction of optical losses among practical applications as they confirm no light scattering at visible or longer wavelengths [48]. The influence of quantum effects on QDs in terms of nonlinear optical property is mainly represented on behalf of increasing polarizability as well as the absorbance index that awards QDs larger nonlinear optical parameters than that of ordinary materials. To make SHG numerical simulation from QDs via applying density matrix, Cox et al [109] found that adjusting the electric field intensity intensified SHG signal. Thus, such types of QDs can be utilized to produce NLO nano switches. Electronic structures that have electrons or holes restricting movement in three dimensions lead to energy level separation, yields quantum confinement, superficial and nonlinear effects [84], and reveal various signs of progress among diverse areas such as sensors, medicines, photoelectric materials, and solar energy batteries [109-113]. 
Quantum dots nonlinear optical properties depend upon their shape, sizes, and compositions. H. Pan et al. reveal NLO properties of colloidal CdSe/ZnS QDs of various concentrations using the Z-scan technique plus nanosecond pulse laser in NIR spectral band to observe QDs concentration with excitation pump energy impact over $\mathrm{CdSe} / \mathrm{ZnS}$ QDs NLO properties. Outcomes of H. Pan et al. represent saturable absorption (SA) behavior of particular core-shell QDs: symmetrical peaks of each obtained normalized transmission curve signify nonlinear SA among samples. The nonlinear absorption effect becomes stronger via increasing QDs concentration, which shows that NLO responses and strong quantum confinement were observed simultaneously [114]. For instance, $\mathrm{Du}$ et al controlled $C d_{x} Z n_{1-x} S e$ chemical components of three QDs and composite $C d_{x} Z n_{1-x} S e$ QDs with various elements proportions. Through Z-scanning, they investigate specific QDs NLO properties and establish that by increasing the amount of $\mathrm{Cd}$ component, the nonlinear absorption coefficient increases from $8.63 \times 10^{-11} \mathrm{~m} / \mathrm{w}$ to $1.31 \times 10^{-10} \mathrm{~m} / \mathrm{w}$ and nonlinear refractivity increases from $-3.06 \times 10^{-11}$ esu to. $-8.56 \times 10^{-11}$ esu.

Core-shell QDs with improved properties are highly functional materials and their properties can be modified via varying the core to shell ratio [115]. In a review article by Karele et al [116], various coreshell QDs applications have been summarized. Core-shell QDs have improved third-order susceptibilities as compared with bulk material structures [117]. Different applications of QDs third-order NLO effects have been examined in photonic devices and optoelectronic fields. Nanoparticle colloidal solutions inserted among various insulator matrices were also considered and established many potential applications, including as optical sensors, in the pharmaceutical industry, drug delivery, in industrial products, imaging ascribed to their optical, electrical, and thermal components, and high electrical conductivity and biological properties due to their surface to volume ratio. The development of optical gain in quantum dots is the main step toward quantum dot lasers. Lasing in quantum dots results in large optical gain at the wavelength of the emitting transitions. At wavelengths that are tunable with the size of quantum dots, narrowband stimulated emission along with a pronounced gain threshold was observed [83]. Many individual reports from various researchers also illustrate the fact that core-shell QDs are gradually attracting more and more attention from technology fields and are emerging as an important part in many fields, such as nonlinear optics [83, 118], electronic engineering [119], and enhancing optical properties [120-122], because quantum dots can control nonlinear optical properties and produce large optical nonlinearities through varying sizes.

In nonlinear microscopy, quantum dots are broadly used as photostable emitters as particular quantum dots emit two-photon luminescence. The study by D. Chauvat et al. shows that in $\mathrm{CdTe} /$ $\mathrm{CdS}$ core-shell quantum dots $(10-15 \mathrm{~nm}$ diameter) secondharmonic generation was obtained. The excitation spectrum of particular quantum dots shows resonance in nonlinear efficiency with an overall maximum at $970 \mathrm{~nm}$. Exploration of Polarization of second harmonic emission confirms the zinc blende symmetry and allows the extraction (3-dimensional) of quantum dots orientation. The quantum dots small size along with orientation sensitivity and intrinsic coherence of SHG process were well adapted for orientation tracking for bioimaging applications and for ultrafast probing of optical near-fields with high resolutions [65]. The future generation of core-shell QDs will exhibit many new properties that will surely result in new applications with improved performance.

\section{CdSe-CdTe TYPE-II CORE-SHELL QUANTUM DOTS NONLINEAR OPTICAL PROPERTIES}

Electrons and holes of type-II core-shell QDs remain in different regions that will cause an active bandgap in the material. $M$. Çadirci, Y. Gündoğdu, E. Elibol et al. [123] depict seven CdSe/ CdTe core-shell type-II QDs of various shell thicknesses as well as different core sizes with different NLO behavior (nonlinear absorption third-order nonlinear susceptibility $\chi^{3}$ to be about $10^{-12}$ esu) via using femtosecond laser with 90 fs pulse at $1 \mathrm{kHz}$ repetition rate at $800 \mathrm{~nm}$ wavelength. To determine the nonlinear parameters, including Kerr coefficient $\left(n_{2} \sim 10^{-20} \mathrm{~cm}^{-2} / W\right)$ and two-photon absorption coefficient $\left(\beta \sim 10^{-20} \mathrm{~cm}^{-2} / W\right)$, open aperture and closed aperture Z-scan measurements were utilized to find $\chi^{3}$. Two unlike CdSe cores with dissimilar CdTe shell thicknesses were utilized in this particular study and their properties were studied and CdSe core sizes beside CdTe shell thickness were assessed through theoretical formulas specified in Refs. [124, 125]. CdSe/CdTe core-shell type-II QDs calculated $n_{2}$ and $\beta$ parameters were displayed in Table.2.

$$
\begin{gathered}
\left|\chi^{(3)}\right|=\left\{\left[\operatorname{Re}\left(\chi^{(3)}\right)\right]^{2}+\left[\operatorname{Im}\left(\chi^{(3)}\right)\right]^{2}\right\}^{1 / 2} \\
\operatorname{Re} \chi^{(3)}=10^{-4} \frac{\varepsilon_{0} n^{2} c^{2}}{\pi} n_{2} \\
\operatorname{Im} \chi^{(3)}=10^{-2} \frac{\varepsilon_{0} c^{2} n_{0}^{2} \lambda}{4 \pi^{2}} \beta
\end{gathered}
$$

CdSe/CdTe core-shell quantum dots $\chi^{(3)}$ have been found out via calculating values of $n_{2}$ and $\beta$ using equation 3 [127, 128]. Particular observation demonstrates that $\mathrm{CdSe} / \mathrm{CdTe}$ core-shell quantum dots nonlinear optical parameters were consistent and $\chi^{(3)}$ have identical values for S1.1 and S1.2. Samples and values of $\chi^{(3)}$ for samples S2.1, S2.2, and S2.3 were regularly increased with the increase in the shell thickness and CdSe/CdTe type-II QDs third-order susceptibility has been observed about $10^{-12}$ esu respectively [123].

\section{HIGHLY LUMINESCENT CdSe CORE- SHELL QUANTUM DOTS NONLINEAR OPTICAL APPLICATIONS}

Ultra-small QDs such as CdSe QDs display saturable absorption (SA) and reverse saturable absorption (RSA) NLO absorption properties for $5 \mathrm{~ns}$ pulses of $532 \mathrm{~nm}$ laser depending upon their compositions, size, and architectures. An effective approach to tuning CdSe-based QDs nonlinear absorption mechanism is by applying core-shell architectures. Core-shell architecture increases the nonlinear absorption as associated with particular distinct parts for local field enhancement related to core-shell structures. As input intensity of CdSe QDs rises they 
TABLE 2 | List of samples with calculated parameters $\beta, n_{2}$, and $\chi^{3}$ by using fs laser z/scan technique [123].

\begin{tabular}{|c|c|c|c|c|c|c|c|c|}
\hline \multirow[t]{2}{*}{ Sample number } & \multirow[t]{2}{*}{ Structure type } & \multicolumn{2}{|c|}{ Composition } & \multicolumn{2}{|c|}{ Size (nm) } & \multirow[t]{2}{*}{$\beta\left(\mathbf{c m}^{2} / \mathbf{W}\right)$} & \multirow[t]{2}{*}{$\mathrm{n}_{2}\left(\mathrm{~cm}^{2} / \mathbf{W}\right)$} & \multirow[t]{2}{*}{$x^{3}$} \\
\hline & & Core & Shell & Core & Shell & & & \\
\hline S1 & Type-I & CdSe & - & $2.84 \mathrm{~nm}$ & - & $-7.618 \times 10^{-12}$ & $-6.3320 \times 10^{-20}$ & $2.6156 \times 10^{-12}$ \\
\hline S1.1 & Type-II & CaSe & CdTe & $2.84 \mathrm{~nm}$ & $0.9 \mathrm{~nm}$ & $-9.053 \times 10^{-12}$ & $-2.3722 \times 10^{-20}$ & $3.1083 \times 10^{-12}$ \\
\hline $\mathrm{S} 1.2$ & Type-II & CdSe & CdTe & $2.84 \mathrm{~nm}$ & $1.15 \mathrm{~nm}$ & $-8.764 \times 10^{-12}$ & $-4.3023 \times 10^{-20}$ & $3.0091 \times 10^{-12}$ \\
\hline S2 & Type-I & CaSe & - & $3.04 \mathrm{~nm}$ & - & $-8.1 \times 10^{-12}$ & $-3.0627 \times 10^{-20}$ & $2.7811 \times 10^{-12}$ \\
\hline S2.1 & Type-II & CaSe & CdTe & $3.04 \mathrm{~nm}$ & $0.7 \mathrm{~nm}$ & $-4.05 \times 10^{-12}$ & $-5.1448 \times 10^{-20}$ & $1.390 \times 10^{-12}$ \\
\hline $\mathrm{S} 2.2$ & Type-II & CdSe & CdTe & $3.04 \mathrm{~nm}$ & $1.07 \mathrm{~nm}$ & $-7.168 \times 10^{-12}$ & $-4.9537 \times 10^{-20}$ & $2.4611 \times 10^{-12}$ \\
\hline $\mathrm{S} 2.3$ & Type-II & CdSe & CdTe & $3.04 \mathrm{~nm}$ & $1.16 \mathrm{~nm}$ & $-9.932 \times 10^{-12}$ & $-4.3012 \times 10^{-20}$ & $3.4101 \times 10^{-12}$ \\
\hline
\end{tabular}
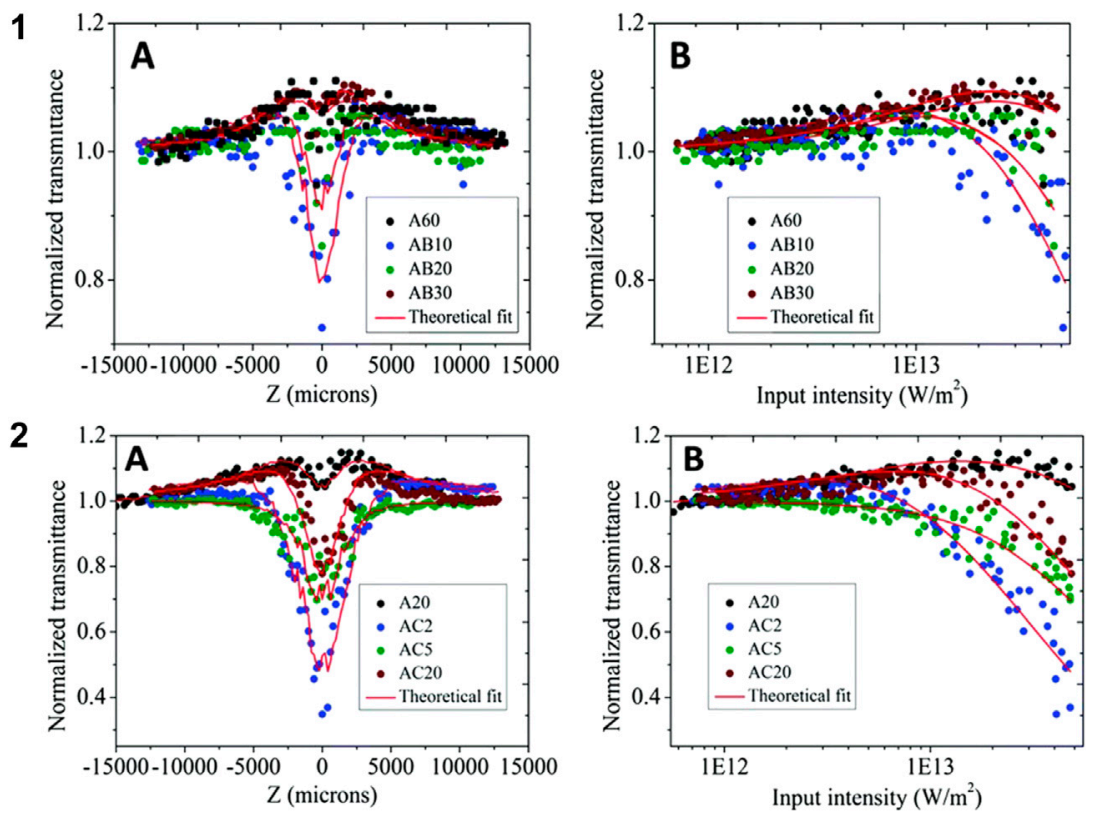

FIGURE 9 | (1) Various shell thickness CdSe/ZnS core-shell QDs measured curves, (2) Various shell thickness CdSe/CdS core-shell QDs measured curves (In figure legends thickness increases from top to bottom): (A) Z-scan open aperture curves. (B) Normalized optical transmission curves. Experimental data was shown by symbols while solid curves display numerical fits calculated via using the absorption coefficient equation [128]. "Republished with permission of the Royal Society of Chemistry, from Ref. [128], copyright 2020; permission conveyed through Copyright Clearance Center, Inc."

display SA or RSA phenomenon and particular nanostructured materials are of great use as saturable absorbers and optical limiters candidates [128].

Studies by S. Bhagyaraj et al. [128] describe nonlinear optical absorption of CdSe QDs by the Z-scan open aperture technique. Particular QDs possess strong nonlinear light transmission and, depending upon QDs size, particular QDs exhibits third-order NLO phenomenon such as SA and RSA. In particular research, core-shell QDs (CdSe-CdS and CdSe-ZnS) exhibit nonlinear absorption behavior independent of shell materials. However, the nonlinear absorption behavior could be influenced by shell thickness, as shown in Figure 9 (1)A,B, (2)A,B.

The above comparisons show that nonlinear absorption is a maximum for thin shells and further nonlinear absorption saturation can be observed with increment in the shell thickness, which makes the material act as a saturable absorber. Local fields associated with individual counterparts are the reasons for the nonlinear absorption enhancement for the core-shell structures QDs [129]. Core-shell structures' shell thickness effects and the QDs' size-dependent nonlinear absorption effects are shown in Figure 10. These results show that core-shell architecture was suggested and such QDs can be used as saturable absorbers and optical limiters.

\section{TWO-PHOTON ABSORPTION OF SEMICONDUCTOR QUANTUM DOTS (QDS) DEPENDING UPON SIZE}

The quantum confinement effect plays an important role in the optical properties of semiconductor quantum dots (QDs) 

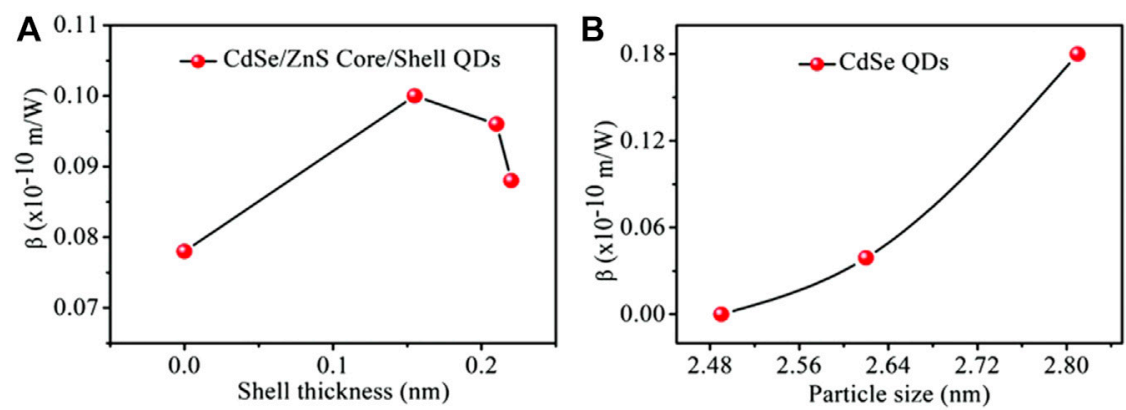

FIGURE 10| Nonlinear absorption coefficient variation as a function: (A) shell thickness and (B) QD size [128]. "Republished with permission of the Royal Society of Chemistry, from Ref. [128], copyright 2020; permission conveyed through Copyright Clearance Center, Inc."
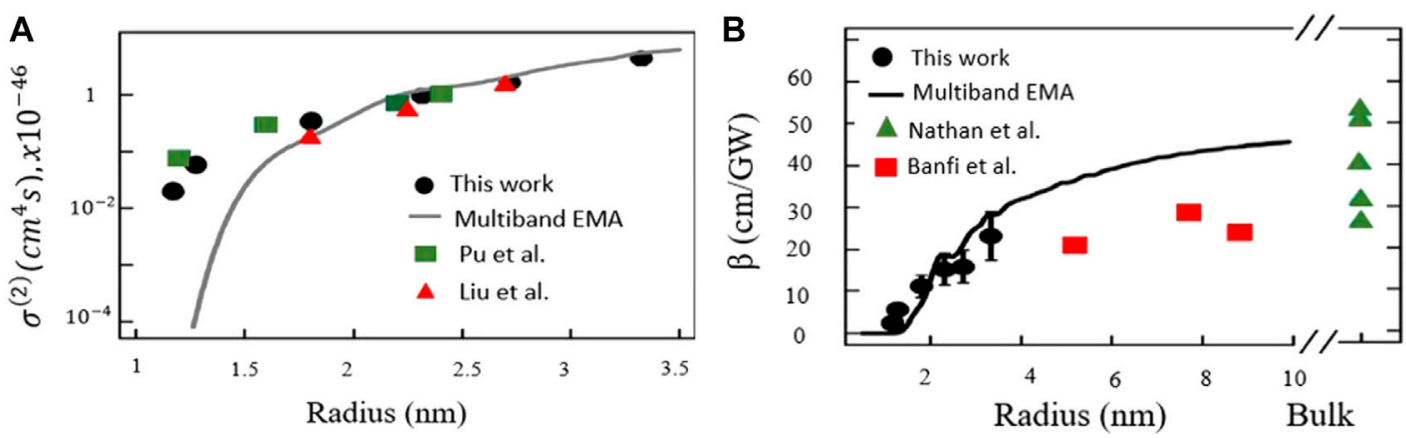

FIGURE 11 | (A) Two-photon absorption cross-section $\sigma^{(2)}$ dependence on CdSe QD radius at 800 nm: simulation based on a multiband EMA (solid line) and experimental values (black circles). Earlier studies results from Pu et al. [130] (green square) and Liu et al. [131] (red triangles) were included for comparison. (B) Twophoton absorption cross-section $\beta$ dependence on CdSe QD radius at $800 \mathrm{~nm}$ : experimental values (black circles), simulation (solid line). Earlier studies result from Ref. [132] on $\mathrm{CdS}_{x} \mathrm{Se}_{1-x}$ nanocrystals in glass and (red square) and from Ref. [133] $\beta$ for bulk CdSe at $800 \mathrm{~nm}$ (green triangles) were included for comparison.

and serves as the basis for the QDs applications in optoelectronic devices and imaging. G. L. Dakovski, J. Shan et al. have studied degenerate two-photon absorption (TPA) (a third-order nonlinear optical process) of below band-gap radiation in CdSe QDs depending upon size. In this work, results from experiment and modeling have been combined for systematical study. Two-photon absorption coefficient $\beta$ of CdSe QDs in a wide range of sizes $(\mathrm{R}=$ 1.1-3.4 nm) has been investigated by femtosecond white transient absorption spectroscopy via probing the pump induced bleaching at the first exciton transition energy at $800 \mathrm{~nm}$.

TPA coefficient $\beta$ and TPA cross-section $\sigma^{(2)}$ has been extracted accordingly for CdSe QDs of varying radii from experimental measurements. The results have been summarized in the below figures and compared with the simulation-based on multiband effective mass approximation (EMA) (solid line) and with some available literature on either $\sigma^{(2)}$ and $\beta$ for CdSe QDs and bulk materials. For $\sigma^{(2)}$ and $\beta$ (black circles) respectively, the reproducibility and uncertainties on the QD concentration were reflected by vertical error bars.

In Figure 11A, increases in QDs radius depict the rise $\sigma^{2}$ and earlier results show excellent agreement with $\sigma^{2}$ on smaller CdSe QDs. In Figure 11B the QDs TPA properties depending upon size however can be observed in TPA coefficient $\beta$. G. Banfi et al. results for QDs ( $\mathrm{R} \sim 5 \mathrm{~nm}$ ) depict that the TPA coefficient $\beta$ is nearly size-dependent while $\beta$ increases for QDs having radii of $1-3.5 \mathrm{~nm}$. So, an excellent agreement has been obtained that TPA coefficient $\beta$ increases as the size of QDs increase from 1.1 to $3.4 \mathrm{~nm}$ (the strong confinement regime) but TPA coefficient saturates for the bulk CdSe radius as no strong confinement is present in such a case [134].

\section{QUANTUM DOTS (QDs) NONLINEAR OPTICAL SWITCHING AND OPTICAL LIMITING PROPERTIES}

Quantum confinement effects make quantum dots a promising class for third-order nonlinear optical materials. In recent years, CdSe QDs, mainly due to their size-dependent luminescence and nonlinear optical properties, have received a great deal of interest. Quantum dots nonlinear optical switching and optical limiting are of great importance. A. Patra et al. in their studies highlighted the nonlinear optical switching and optical limiting of CdSe QDs via nanosecond Z-scan measurement at $532 \mathrm{~nm}$ ns pulse laser. These particular works represent that saturable absorption (SA) of nonlinear processes dominates at lower intensities while at 


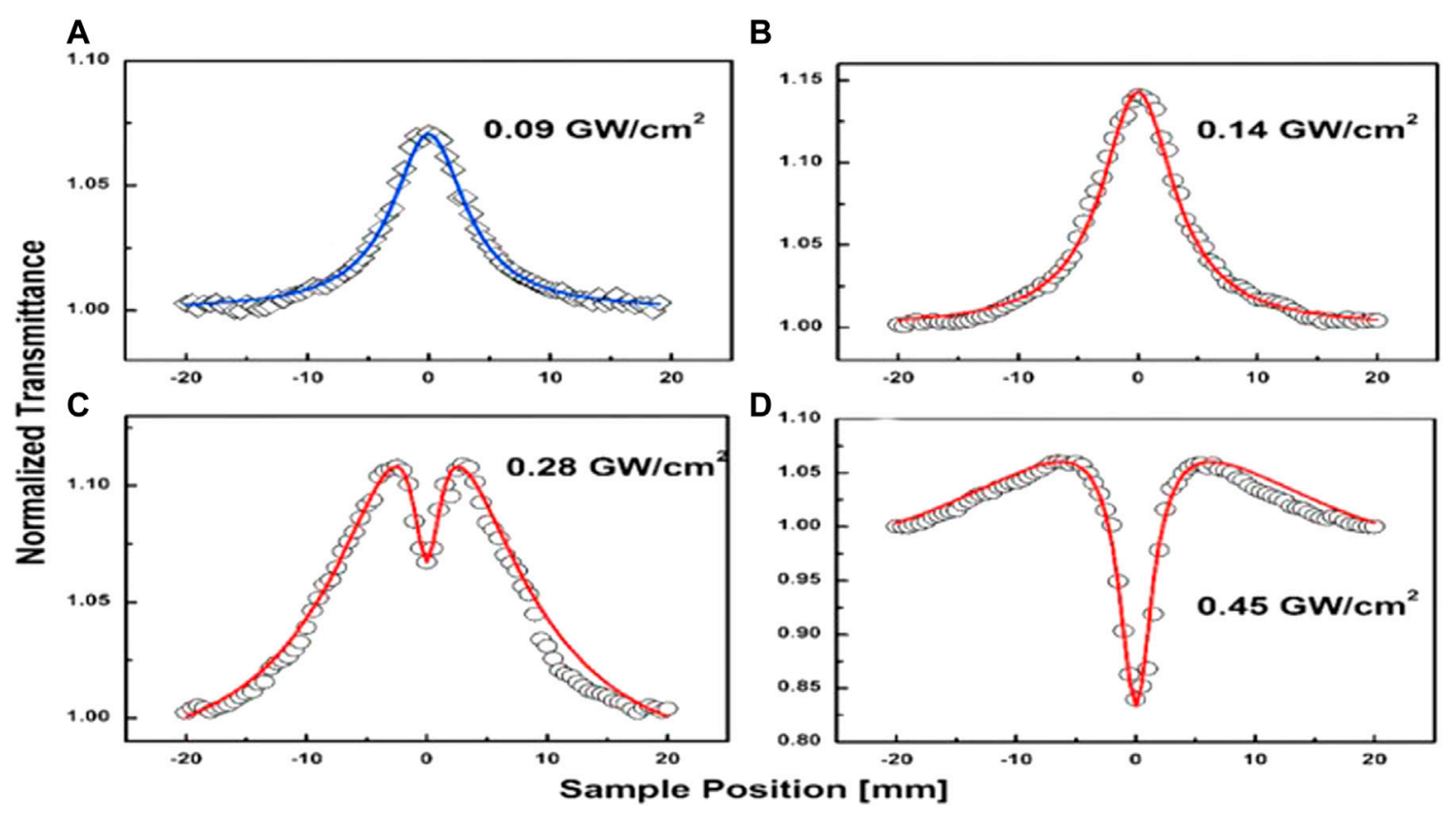

FIGURE 12 | CdSe QDs intensity-dependent nonlinear absorption curves at various input intensities with $532 \mathrm{~nm}$, ns laser pulse [135].

higher intensities it changes to reverse saturable absorption (RSA). The saturable absorption process is attributed to the ground state bleaching and reverses saturation absorption is ascribed to free carrier absorption (FCA) of CdSe QDs. Such behavior of CdSe QDs makes them a suitable candidate for optical devices and optical limiting applications.

Figure 12 represents the intensity-dependent CdSe QDs open aperture Z-scan curves with $532 \mathrm{~nm}$ at input intensities from $0.09 \mathrm{GW} / \mathrm{cm}^{2}$ to $0.45 \mathrm{GW} / \mathrm{cm}^{2}$. These figures represent that when the intensity value is increased from $0.09 \mathrm{GW} / \mathrm{cm}^{2}$ to $0.14 \mathrm{GW} / \mathrm{cm}^{2}$ the saturable absorption becomes stronger while the Z-scan curve broadens. Further increasing the intensity value, the transmittance decreases resulting in reverse saturable absorption (RSA). In this case, the resonant nonlinear absorption process dominates via taking excited electron of conduction band to higher conduction band states through the second photon. This process is known as free carrier absorption (FCA) which results in reverse saturable absorption.

At higher intensities, the saturable absorption of lower excited states and free-carrier absorption compete with each other, resulting in reverse saturable absorption over saturable absorption. The free carrier absorption and thermal nonlinearities are responsible for optical limiting behavior in nanosecond lasers. The present investigation of CdSe QDs could be useful for mode-locking laser systems as saturable absorption is obtained at lower intensities and can work as an optical limiter based on RSA behavior at high intensities. However, this behavior of saturable absorption over reverse saturable absorption can be utilized in optical pulse compressor, optical switching, and optical pulse narrowing. These results show various applications in optical-based limiting devices. If we compare the pico-second (Ref. [136]) and nanosecond laser, results clearly show that in the case of open aperture Z-scan results, a similar switch of SA to RSA is observed. However, the nonlinear absorption involved free carrier absorption under nanosecond laser excitation whereas direct two-photon absorption and free-carrier absorption/excited state absorption dominate. In the case of nanosecond laser excitation, thermal nonlinearities arise while in picosecond laser excitation electronic contribution arises. One point is also noticeable at this level that signs of nonlinearities indicate their origin as thermal nonlinearities constitutes a negative sign while electronic contribution constitutes positive nonlinearity. The optical nonlinearities in nanosecond laser excitation are always higher than that in pico-second laser excitations and their difference could be due to the pulse duration effects [135].

\section{QUANTUM DOTS (QDS) SIZE-DEPENDENT MULTIPHOTON ABSORPTION AND REFRACTION PROPERTIES}

With direct bandgap and control on the bandgap over a spectral range, QDs exhibits many applications in the fields of optoelectronics and nano-photonics. CdSe QDs are by far the most studied QDs among all quantum dots. Nonlinear optical properties of CdSe QDs were studied by Venkatram et al. with $800 \mathrm{~nm}$ wavelength and 110 femtosecond laser. Various sizes of CdSe QDs were utilized such as $5 \mathrm{~nm}, 10 \mathrm{~nm}$, $25 \mathrm{~nm}, 400 \mathrm{~nm}$. Figure 13A shows how a decrease in the size of QDs causes the shift of absorption peak toward the shorter wavelengths. Results depict that $5 \mathrm{~nm}$ and $10 \mathrm{~nm}$ CdSe QDs exhibit four-photon absorption (4 PA) while $20 \mathrm{~nm}$ and $400 \mathrm{~nm}$ CdSe QDs exhibit threephoton absorption (3 PA). Based on these studies, experimental results of open aperture Z-can measurements were compared with the multiphoton absorption coefficients in Figure 13B,C. 

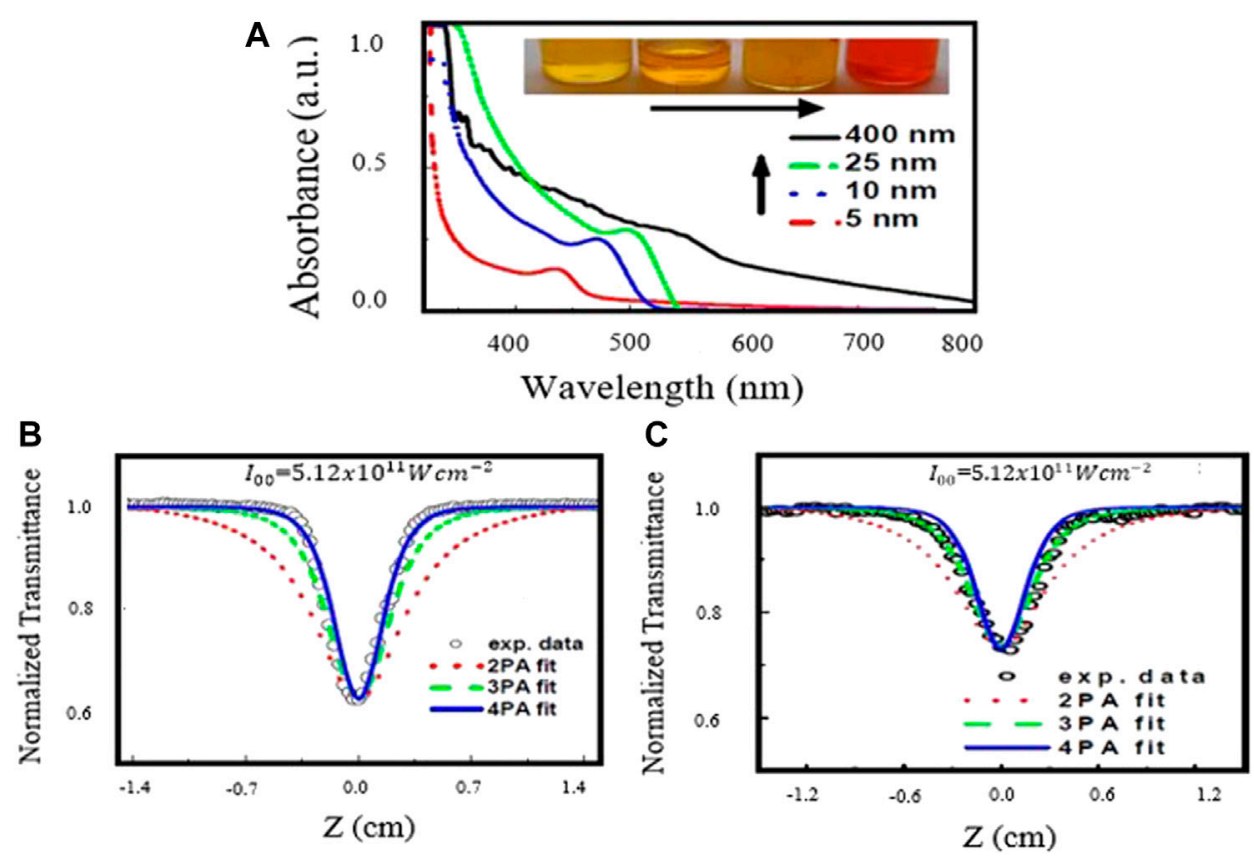

FIGURE 13 | (A) CdSe QDs Optical absorption spectra of various sizes such as $5 \mathrm{~nm}, 10 \mathrm{~nm}, 20 \mathrm{~nm}, 400 \mathrm{~nm}$ (B) CdSe QDs (5 nm) open aperture Z-scan curve. (C) Open aperture Z-scan curve of $400 \mathrm{~nm}$ CdSe QDs. Lines show the theoretical fit with multiphoton absorption coefficients [137].

Figure 13B represents that $2 \mathrm{PA}$ and $3 \mathrm{PA}$ fitted data deviates from the experimental data for $5 \mathrm{~nm}$ CdSe QDs while $4 \mathrm{PA}$ data fits well with the experimental data. Similar results were obtained for $10 \mathrm{~nm}$ CdSe QDs. For $25 \mathrm{~nm}$ and $400 \mathrm{~nm}$, Figure 13C shows that 3 PA fits well as compared to the $2 \mathrm{PA}$ and $4 \mathrm{PA}$ [137]. These results show that by changing the sizes of QDs nonlinear optical effects also change. A change in size causes quantum confinement effects and, due to these effects, nonlinear optical properties among various quantum dots are enhanced and can be utilized for beneficial purposes.

\section{USES OF CORE-SHELL QUANUTM DOTS AS OPTICAL GAIN AND LASING APPLICATIONS}

In quantum dots, development of optical gain becomes an important step to achieve quantum dot lasers. Studies by V. I. Klimov et al. represent that large optical gain can be achieved as a result of lasing in quantum dots at the wavelengths of the emitting transitions. Narrowband stimulated emission with an increased gain threshold can be achieved at wavelengths that are tunable with the size of quantum dots as expected from quantum confinement effects [83]. Quantum dots, consisting of between 100 and 10,000 atoms, can now be easily synthesized based on their various properties such as their size $(1-10 \mathrm{~nm})$, shape (spherical, elongated, hollow, branched, etc.), and composition (IV, II-VI, I-III-VI, I-II-VI-VI grouped materials). As described earlier, core-shell QDs exhibit favorable optical properties like high absorption cross-section, quantum yields, high photostability, and high optical gain for developing QDs-based microlasers. They are interesting candidates for lasers as active materials as their surface chemistry can be modified and their structures can be precisely controlled, which allows for their efficient inclusion into solid films. Colloidal nanocrystals have been extensively studied for the realization of lasing media with broadly tunable emission wavelengths. The main property for a lasing application that has to be boosted up is an optical gain that is defined as the difference between the absorption and stimulated emission rates.

High performance and extremely stable optical gain media have been obtained for tailored CdSe core (spherical) and CdS shell (rod) QDs via one- and two-photon pumping. Using CdSe/CdS nanorod heterostructures, lasing under one and two-photon pumping has been obtained by using CdSe/CdS nanorod heterostructures that comprise a spherical CdS core $(2.4 \mathrm{~nm}$ diameter) encapsulated in a rod-like shell $(39 \mathrm{~nm})$ as the optical gain media. The main advantage of this optical configuration is to realize the material variable emission wavelength (emission can be tuned by changing the physical dimension of shell and core). For $39 \mathrm{~nm}$ long nanorods, a two-photon absorption cross-section obtained value is $2.3 \times 10^{-5} \mathrm{GM}$ which is $2-4$ times larger in magnitude as compared to the previously reported core-shell QDs (both core and shell are spherical).

Importantly, two-photon absorption cross-section can be adjusted independently for the CdSe core dimensions, whose size primarily determine the emission wavelength. These results depict that by changing the composition and dimensions of core-shell quantum dots, a nonlinear phenomenon that has a two-photon absorption cross-section can be achieved of greater value as compared to spherical core-shell QDs. Based on these results, particular 
TABLE 3 | Various applications of quantum dots (QDs) in different fields.

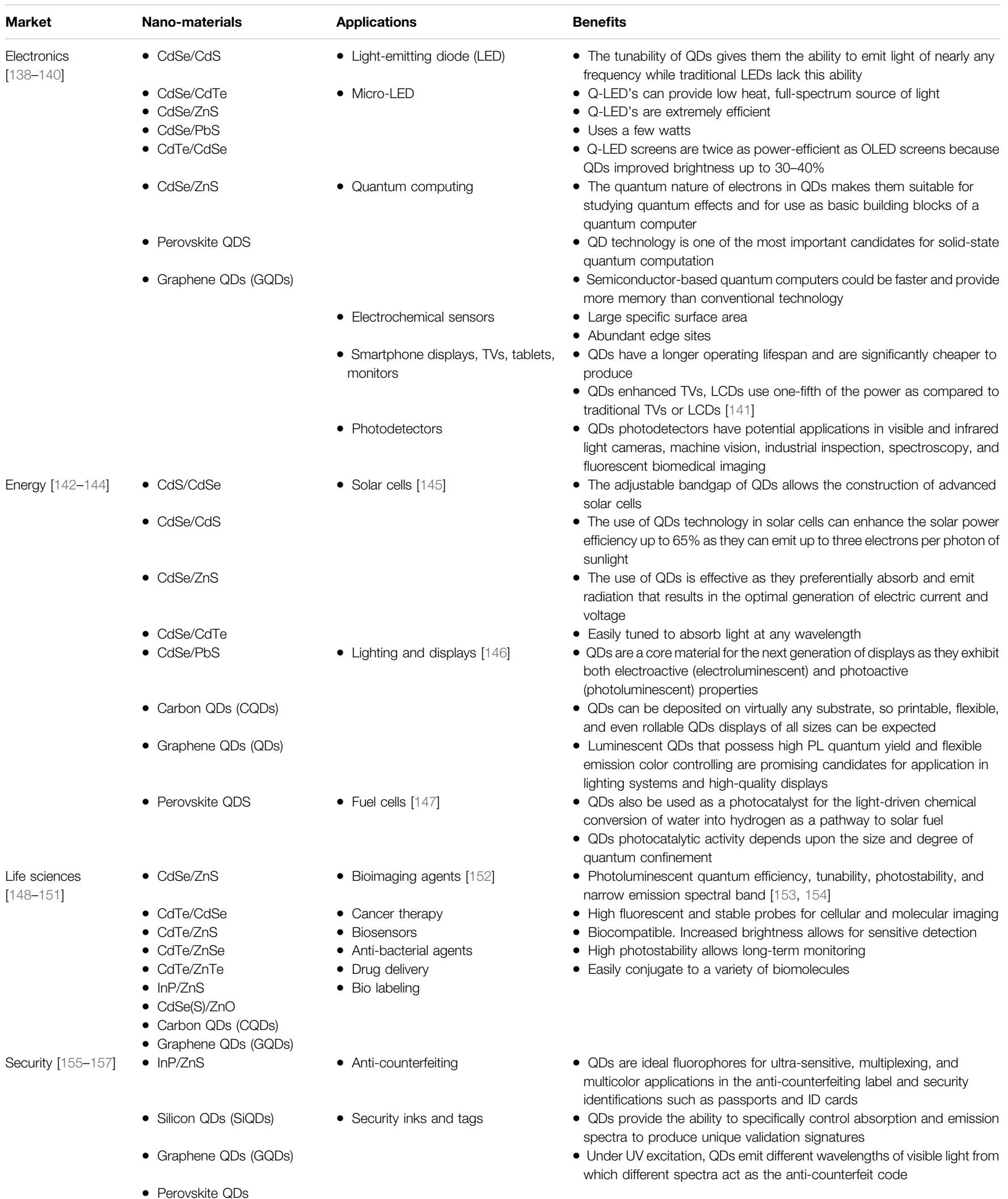


TABLE 3 | (Continued) Various applications of quantum dots (QDs) in different fields.

\begin{tabular}{|c|c|c|c|}
\hline Market & Nano-materials & Applications & Benefits \\
\hline Batteries [158] & - Graphene QDs (GQDs) & $\begin{array}{l}\text { - Anodes, cathodes, and electrolytes } \\
\text { in Li-ion batteries }\end{array}$ & $\begin{array}{l}\text { - Improved cycling and storage capacities [159] } \\
\text { - Can withstand thousands of charge/discharge cycles } \\
\text { - Prolonging battery life }\end{array}$ \\
\hline Photonics [140] & - Perovskite QDs & - Lasers & - Utilizes index coupling grating on GaAs material systems \\
\hline Sensors [140, & - CdSe & - DNA biosensing & - Size adjustable, bright fluorescence \\
\hline \multirow[t]{5}{*}{$160,161]$} & - CdTe & - Glucose biosensors & $\begin{array}{l}\text { - Narrowband edge luminescence, strong resistance against } \\
\text { photobleaching }\end{array}$ \\
\hline & - $\mathrm{CdSe} / \mathrm{ZnS}$ & - Food safety detection & - Excellent compatibility with biological molecules [162] \\
\hline & - Carbon QDs (CQDs) & - Drug discovery & \\
\hline & - Graphene QDs (GQDs) & - Detection of pathogens & \\
\hline & $\begin{array}{l}\text { - Cadmium-free QDs } \\
\text { (CFQDs) }\end{array}$ & - Pesticide detection & \\
\hline Thermoelectric [163] & $\begin{array}{l}\text { - } \text { Colloidal QDs } \\
\text { - } \text { Quantum dot superlattice } \\
\text { structures (QLSL) } \\
\text { - InAs/InP } \\
\text { - } \mathrm{PbTe/PbSeTe} \mathrm{QLSL}\end{array}$ & - Cooling and waste heat recovery & $\begin{array}{l}\text { - Can potentially boost the energy conversion efficiency from } 20 \text { to } 30 \% \\
\text { depending upon temperatures }\end{array}$ \\
\hline
\end{tabular}

core-shell QDs can be used for various optical devices. The size-dependent advantages afforded by quantum confined CdSe core are therefore preserved for heterostructures. Nanorods have also been used as a gain medium for twophoton pumped lasing using a spherical optical cavity. Smart Engineering of heterostructures, known as core-shell QDs, provides exciting opportunities for applications in energy technologies [50]. The usefulness and application of Quantum Dot technology continues to expand and research is striving to bring their benefits to more and more technologically applied fields. Quantum dots have extraordinary potential applications with noticeable results in various fields of sciences, such as those shown in Table 3.

Core-shell QDs are a central focus in nanotechnology and are expressively used for the manufacturing of Nanodevices. To achieve higher quantum yield (QY), photoluminescence (PL), and upgraded nonlinearities, a strong confinement regime is necessary. Low dimensional materials, such as QDs and coreshell QDs, have been extensively studied and significant progress has been made, particularly in II-VI group compounds such as CdSe and CdTe due to high photoluminescence (PL), good quantum yield (QY), and optical nonlinearities. Efros and Brus gave the first theoretical descriptions of three-dimensional excitonic quantum confinement. These authors discussed the basic optical properties of quantum dots for different quantum confinement regimes [164]. Reiss et al. [165] reported a new method for the preparation of core-shell semiconductor nanocrystals of the A (II) B (VI) family. The photoluminescence spectra were measured and a significant increase in the fluorescence intensity of the CdSe/ZnS and $\mathrm{CdSe} / \mathrm{ZnSe}$ compared to CdSe was observed. The third-order nonlinear optical properties of CdSe/ZnS core-shell quantum dots in solution with toluene were studied over a spectrum ranging from 450 to $680 \mathrm{~nm}$ using 225-fs pulse [166]. For calculating the $1 \mathrm{~s}-1 \mathrm{~s}$ electronic transition in spherically layered semiconductor quantum dots extended theoretical approach was introduced by A. Eychmüller et al. [167]. Venkatram et al. reported that CdSe nanoparticles of different sizes show multiphoton absorption under $800 \mathrm{~nm}$ excitation [168]. The particle size ranging from 4.16 to $5.25 \mathrm{~nm}$ of CdSe QDs exhibits reverse saturable absorption under $532 \mathrm{~nm}$ laser excitation and the two-photon absorption (TPA) coefficient values enhance when decreasing the bandgap [137]. D. Wawrzynczyk et al. reported the large two-photon absorption cross-section of CdSe QDs (QRs) which is of potential use for nanophotonics and bioimaging [169]. The nonlinear optical properties of CdSe QDs with $532 \mathrm{~nm}$ ns laser excitation are compared with $532 \mathrm{~nm}$ ps laser excitation, which is published in Ref. [136]. In the case of open aperture Z-scan results, it is shown that a similar switch over SA to RSA nonlinear optical behavior was observed under nanosecond and picosecond laser excitations. However, the origination of nonlinear absorption involved free carrier absorption under ns laser excitation, whereas under picosecond laser excitations, direct TPA and free-carrier absorption/excited state absorption dominate. In the case of nonlinear refraction, where the self-defocusing effect of the laser beam occurs, thermal nonlinearities dominate in the case of ns laser excitation and electronic contribution dominates during picosecond laser excitation. The optical nonlinearities in nanosecond laser excitation are higher than that of picosecond laser excitation and the difference could be attributed to the pulse duration effects [135]. These effective materials have substantial applications in the future of electronics, medicine, photonics, and optoelectronics [170]. Moreover, QDs indicate favorable progress among solar cells, as they have the capacity of changing absorbed energy to electrical energy [171].

\section{CONCLUSION}

In this review, particular characteristics, including the structure, properties, and applications of low dimensional materials, such as QDs and core-shell QDs, have been discussed. Quantum dots are among the most investigated materials because their optical 
properties can be controlled via their size and a large surface-tovolume ratio provides a large number of surface traps to accelerate the recovery process. Nonlinear QDs with advanced quality can be obtained by manipulating the QDs size, shape, surface, design, and chemical composition. These particular studies have taken into account some significant findings of nonlinear optical properties in CdSe and CdTe QDs coreshell QDs.

Core-shell QDs improved optical properties, including increased quantum yield (QL) and photostability of core QDs, which highlights the necessity of synthesizing core-shell QDs. Using different synthesis approaches to prepare various coreshell QDs has been utilized to observe their NLO properties, such as third-order nonlinear susceptibility $\chi^{3}$ (SA as well as RSA), by using various techniques such as the Z-scan technique. Enhanced third-order nonlinearity was attained via fine controlling over core-shell QDs composition and thickness which in return plays an efficient role in optical data storages and optical switching devices. Different core-shell QDs can be used for different purposes such as optical limiters and saturable absorbers by using different core-shell architecture approaches. By different core-shell QDs architecture designs, core-shell QDs nonlinear optical behavior was enhanced so that it can be used as reliable

\section{REFERENCES}

1. Chon JWM, Gu M, Bullen C, and Mulvaney P. Three-photon excited band edge and trap emission of CdS semiconductor nanocrystals. Appl Phys Lett (2004) 84(22):4472-4. doi:10.1063/1.1755420

2. Wang $\mathrm{Y}$, and Herron N, Nanometer-sized semiconductor clusters: materials synthesis, quantum size effects, and photophysical properties. J Phys Chem. American Chemical Society (1991) 95:525-32. doi:10.1021/j100155a009

3. Eustis S, and El-Sayed MA. Why gold nanoparticles are more precious than pretty gold: noble metal surface plasmon resonance and its enhancement of the radiative and nonradiative properties of nanocrystals of different shapes. Chem Soc Rev (2006) 35(3):209-17. doi:10.1039/b514191e

4. Yu B, Zhu C, and Gan F. Nonlinear optical absorption and refraction by CdTe microcrystals doped in glass. J Appl Phys (2000) 87(4):1759-61. doi:10.1063/ 1.372088

5. Guo L, Yang S, Yang C, Yu P, Wang J, and Ge W. Highly monodisperse polymer-capped $\mathrm{ZnO}$ nanoparticles: preparation and optical properties. Appl Phys Lett (2000) 76(20):2901-3. doi:10.1063/1.126511

6. Prakash GV, Cazzanelli M, Gaburro Z, Pavesi L, Iacona F, and Franzò G. Nonlinear optical properties of silicon nanocrystals grown by plasmaenhanced chemical vapor deposition. J Appl Phys (2002) 91(7):4607-10. doi:10.1063/1.1456241

7. Elim HI, Ji W, Yuwono AH, Xue JM, and Wang J. Ultrafast optical nonlinearity in poly(methylmethacrylate)-TiO2 nanocomposites. Appl Phys Lett (2003) 82(16):2691-3. doi:10.1063/1.1568544

8. Yoffe AD. Low-dimensional systems: quantum size effects and electronic properties of semiconductor microcrystallites (zero-dimensional systems) and some quasi-two-dimensional systems. Adv Phys (1993) 42(2):173-262. doi:10.1080/00018739300101484

9. Yoffe AD. Semiconductor quantum dots and related systems: electronic, optical, luminescence and related properties of low dimensional systems. Adv Phys (2001) 50(1):1-208. doi:10.1080/00018730010006608

10. Banfi G, Degiorgio V, and Ricard D. Nonlinear optical properties of semiconductor nanocrystals. Adv Phys (1998) 47(3):447-510. doi:10.1080/ 000187398243537

11. Zou W, Qiao J, Hu X, Ge X, and Lian H. Synthesis in aqueous solution and characterisation of a new cobalt-doped $\mathrm{ZnS}$ quantum dot as a hybrid saturable absorbers, optical limiters, and so on. With rapid nanotechnology development, QDs became a subject of great interest in biology, drug testifying, functional material, and environmental sciences. Frequent QDs investigation together with evolution provides further advancements in finer device fabrication and quantum efficiency.

\section{AUTHOR CONTRIBUTIONS}

UK drafted the manuscript. RY, JQ, and LL contributed to improvements/corrections and approved the fifinal version.

\section{FUNDING}

This work was supported in part by The National Key Research and Development Program of China (Grant No. 2017YFA0700402), National Natural Science Foundation of China (Grant Nos. 61935012/61525503/61620106016/ 61722508/61961136005), and Shenzhen Science and Technology Funding (Grant Nos. JCYJ20180305124902165/ JCYJ20170412105003520).

ratiometric chemosensor. Anal Chim Acta (2011) 708(1-2):134-40. doi:10. 1016/j.aca.2011.09.044

12. Liu P, Wang Q, and Li X. Studies on CdSe/L-cysteine quantum dots synthesized in aqueous solution for biological labeling. J Phys Chem C (2009) 113(18):7670-6. doi:10.1021/jp901292q

13. Li L, Qian H, and Ren J. "Article in chemical communications,". Cambridge, United Kingdom: pubs.rsc.org (2005). doi:10.1039/b412686f

14. Petruska MA, Malko AV, Voyles PM, and Klimov VI. High-performance, quantum dot nanocomposites for nonlinear optical and optical gain applications. Adv Mater (2003) 15(7-8):610-3. doi:10.1002/adma. 200304450

15. Jia W, Douglas EP, Guo F, and Sun W. Optical limiting of semiconductor nanoparticles for nanosecond laser pulses. Appl Phys Lett (2004) 85(26): 6326-8. doi:10.1063/1.1836871

16. Larson DR, Zipfel WR, Williams RM, Clark SW, Bruchez MP, and Wise FW. Water-soluble quantum dots for multiphoton fluorescence imaging in vivo. Science (2003) 300(5624):1434-6. doi:10.1126/ science. 1083780

17. Michalet X, Pinaud FF, Bentolila LA, Tsay JM, Doose S, and Li JJ. Quantum dots for live cells, in vivo imaging, and diagnostics. Science. American Association for the Advancement of Science (2005) 307:5709538-44. doi:10.1126/science.1104274

18. Loss D, and DiVincenzo DP. Quantum computation with quantum dots. Phys Rev A (1998) 57(1):120-6. doi:10.1103/PhysRevA.57.120

19. Haase MA, Qiu J, DePuydt JM, and Cheng H. Blue-green laser diodes. Appl Phys Lett (1991) 59(11):1272-4. doi:10.1063/1.105472

20. Hines MA, and Guyot-Sionnest P. Synthesis and characterization of strongly luminescing ZnS-capped CdSe nanocrystals. J Phys Chem (1996) 100(2): 468-71. doi:10.1021/jp9530562

21. Dabbousi BO, Rodriguez-Viejo J, Mikulec FV, Heine JR, Mattoussi H, and Ober R. (CdSe)ZnS Core-Shell quantum dots: synthesis and characterization of a size series of highly luminescent nanocrystallites. J Phys Chem B (1997) 101(46):9463-75. doi:10.1021/jp971091y

22. Matijevic E. Preparation and properties of uniform size colloids. Chem Mater (1993) 5(4):412-26. doi:10.1021/cm00028a004

23. Mulvaney P, Giersig M, Ung T, and Liz-Marzán LM. Direct observation of chemical reactions in silica-coated gold and silver nanoparticles. Adv Mater (1997) 9(7):570-5. doi:10.1002/adma.19970090712 
24. Philipse AP, Van Bruggen MPB, and Pathmamanoharan C. Magnetic silica dispersions: preparation and stability of surface-modified silica particles with a magnetic core. Langmuir (1994) 10(1):92-9. doi:10.1021/la00013a014

25. Caruso F, Spasova M, Susha A, Giersig M, and Caruso RA. Magnetic nanocomposite particles and hollow spheres constructed by a sequential layering approach. Chem Mater (2001) 13(1):109-16. doi:10.1021/cm001164h

26. Hanprasopwattana A, Srinivasan S, Sault AG, and Datye AK. Titania coatings on monodisperse silica spheres (characterization using 2-propanol dehydration and TEM). Langmuir (1996) 12(13):3173-9. doi:10.1021/ la950808a

27. Lim SJ, Chon B, Joo T, and Shin SK. Synthesis and characterization of zincblende CdSe-based core/shell nanocrystals and their luminescence in water. J Phys Chem C (2008) 112(6):1744-7. doi:10.1021/jp710648g

28. Zhang W, Chen G, Wang J, Ye BC, and Zhong X. Design and synthesis of highly luminescent near-infrared-emitting water-soluble $\mathrm{CdTe} / \mathrm{CdSe} / \mathrm{ZnS}$ Core/Shell/Shell quantum dots. Inorg Chem (2009) 48(20):9723-31. doi:10.1021/ic9010949

29. Pan H, Chu H, Zhao S, Li Y, and Li D. Nonlinear absorption properties of $\mathrm{CdSe} / \mathrm{ZnS}$ quantum dots and the application: passively Q-switched Nd: GdVO4 laser at $1.06 \mu \mathrm{m}$. Appl Phys Express (2019) 12:022001. doi:10. 7567/1882-0786/aaf789

30. Torchynska T, and Vorobiev Y. Semiconductor II-VI quantum dots with interface states and their biomedical applications. Adv Biomed Eng (2011). doi: $10.5772 / 20628$

31. Nyk M, Wawrzynczyk D, Szeremeta J, and Samoc M. Spectrally resolved sizedependent third-order nonlinear optical properties of colloidal CdSe quantum dots. Appl Phys Lett (2012) 100(4):041102-4. doi:10.1063/1. 3679381

32. Brodsky JL, and Andrews DW, "Accepted muscript accepted muspt," pp. $0-11.2011$.

33. Coe BJ. Switchable nonlinear optical metallochromophores with pyridinium electron acceptor groups. Acc Chem Res (2006) 39(6):383-93. doi:10.1021/ ar050225k

34. Dalton LR, Sullivan PA, and Bale DH. Electric field poled organic electrooptic materials: state of the art and future prospects. Chem Rev (2010) 110(1): 25-55. doi:10.1021/cr9000429

35. Radhakrishnan TP. Molecular structure, symmetry, and shape as design elements in the fabrication of molecular crystals for second harmonic generation and the role of molecules-in-materials. Acc Chem Res (2008) 41(3):367-76. doi:10.1021/ar7002072

36. Willets KA, Nishimura SY, Schuck PJ, Twieg RJ, and Moerner WE. Nonlinear optical chromophores as nanoscale emitters for singlemolecule spectroscopy. Acc Chem Res (2005) 38(7):549-56. doi:10.1021/ ar0401294

37. Ray PC. Size and shape dependent second order nonlinear optical properties of nanomaterials and their application in biological and chemical sensing. Chem Rev (2010) 110(9):5332-65. doi:10.1021/cr900335q

38. Autere A, Jussila H, Dai Y, Wang Y, Lipsanen H, and Sun Z. Nonlinear optics with 2D layered materials. Adv Mater (2018) 30(24):1705963-24. doi:10. 1002/adma.201705963

39. Li R, Dong N, Ren F, Amekura H, Wang J, and Chen F. Nonlinear absorption response correlated to embedded Ag nanoparticles in BGO single crystal: from two-photon to three-photon absorption. Sci Rep (2018) 8. doi:10.1038/ s41598-018-20446-6

40. Fu Y, Ganeev RA, Krishnendu PS, Zhou C, Rao KS, and Guo C. Sizedependent off-resonant nonlinear optical properties of gold nanoparticles and demonstration of efficient optical limiting. Opt Mater Express (2019) 9(3):976. doi:10.1364/ome.9.000976

41. Veisi M, Kazemi SH, and Mahmoudi M. Tunneling-induced optical limiting in quantum dot molecules. Sci Rep (2020) 10(1). doi:10.1038/s41598-02073343-2

42. Yin Y, and Alivisatos AP. Colloidal nanocrystal synthesis and the organicinorganic interface. Nature (2005) 437(7059):664-70. doi:10.1038/ nature 04165

43. Alivisatos AP. Semiconductor clusters, nanocrystals, and quantum dots. Science (1996) 271(5251):933-37. doi:10.1126/science.271.5251.933

44. Brus L. Quantum crystallites and nonlinear optics. Appl Phys A (1991) 53(6): 465-74. doi:10.1007/BF00331535
45. Huang G, Yu D, Zhang J, Zhao M, Zhao D, and Pan M. The preparation and application of the nonlinear optical quantum dots. Sel Pap Chin Soc. Opt. Eng. Conf. held Oct. Novemb. (2017) 10255:102552Q. doi:10.1117/12.2264346

46. Petryayeva E, Algar WR, and Medintz IL. Quantum dots in bioanalysis: a review of applications across various platforms for fluorescence spectroscopy and imaging. Appl Spectrosc (2013) 67(3):215-52. doi:10.1366/12-06948

47. Wu M, Petryayeva E, Medintz IL, and Algar WR. Quan Dots: Appl Biol (2014) Vol. 1199.

48. Mansur HS. Quantum dots and nanocomposites. Wiley Interdiscip Rev Nanomed Nanobiotechnol (2010) 2(2):113-29. doi:10.1002/wnan.78

49. Ning T, Gao P, Wang W, Lu H, Fu W, and Zhou Y. Nonlinear optical properties of composite films consisting of multi-armed CdS nanorods and ZnO. Opt Mater (2009) 31(6):931-5. doi:10.1016/j.optmat.2008.10.041

50. Todescato F, Fortunati I, Minotto A, Signorini R, Jasieniak JJ, and Bozio R, Engineering of semiconductor nanocrystals for light emitting applications. Materials (Basel). Basel, Switzerland: MDPI AG (2016) 9(8):672. doi:10.3390/ ma9080672

51. Li J, Fu J, Yang Q, Wang L, Wang X, and Chen L. Thermosensitive molecularly imprinted core-shell $\mathrm{CdTe}$ quantum dots as a ratiometric fluorescence nanosensor for phycocyanin recognition and detection in seawater. Analyst (2018) 143(15):3570-8. doi:10.1039/c8an00811f

52. Yang Q, Li J, Wang X, Peng H, Xiong H, and Chen L. Strategies of molecular imprinting-based fluorescence sensors for chemical and biological analysis. Biosens Bioelectron. Elsevier (2018) 112:54-71. doi:10.1016/j.bios.2018.04.028

53. Wang X, Yu S, Liu W, Fu L, Wang Y, and Li J. Molecular imprinting based hybrid ratiometric fluorescence sensor for the visual determination of bovine hemoglobin. ACS Sens. (2018) 3(2):378-85. doi:10.1021/acssensors.7b00804

54. Reshma VG, and Mohanan PV. Quantum dots: applications and safety consequences. J. Lumin. (2019) 205:287-98. doi:10.1016/j.jlumin.2018.09.015

55. Danek M, Jensen KF, Murray CB, and Bawendi MG. Synthesis of luminescent thin-film CdSe/ZnSe quantum dot composites using CdSe quantum dots passivated with an overlayer of ZnSe. Chem Mater (1996) 8(1):173-80. doi:10. $1021 / \mathrm{cm} 9503137$

56. Rosenthal SJ, McBride J, Pennycook SJ, and Feldman LC. Synthesis, surface studies, composition and structural characterization of CdSe, core/shell, and biologically active nanocrystals. Surf Sci Rep (2007) 62(4):111-57. doi:10. 1016/j.surfrep.2007.02.001

57. Rosenthal SJ, Tomlinson I, Adkins EM, Schroeter S, Adams S, and Swafford L. Targeting cell surface receptors with ligand-conjugated nanocrystals. J Am Chem Soc (2002) 124(17):4586-94. doi:10.1021/ja003486s

58. Pan L, Tamai N, Kamada K, and Deki S. Nonlinear optical properties of thiolcapped CdTe quantum dots in nonresonant region. Appl Phys Lett (2007) 91(5):051902. doi:10.1063/1.2761494

59. Padilha L, Fu J, Hagan D, Van Stryland E, Cesar C, and Barbosa L. Twophoton absorption in CdTe quantum dots. Opt Express (2005) 13(17):6460. doi:10.1364/opex.13.006460

60. Umar AA, Reshak AH, Oyama M, and Plucinski KJ. Fluorescent and nonlinear optical features of CdTe quantum dots. J Mater Sci Mater Electron (2012) 23(2):546-50. doi:10.1007/s10854-011-0434-6

61. Dancus I, Vlad VI, Petris A, Gaponik N, Lesnyak V, and Eychmüller A. Saturated near-resonant refractive optical nonlinearity in CdTe quantum dots. Opt Lett (2010) 35(7):1079. doi:10.1364/ol.35.001079

62. Duhan JS, Kumar R, Kumar N, Kaur P, Nehra K, and Duhan S. Nanotechnology: the new perspective in precision agriculture. Biotechnol Rep (2017) 15:11-23. doi:10.1016/j.btre.2017.03.002

63. Yao J, Li P, Li L, and Yang M. Biochemistry and biomedicine of quantum dots: from biodetection to bioimaging, drug discovery, diagnostics, and therapy. Acta Biomater (2018) 74:36-55. doi:10.1016/j.actbio.2018.05.004

64. Pourjafari S, Molaei M, Saievar-Iranizad E, Dehghani Z, and Majlesara MH. Investigation of the photoluminescence properties and nonlinear optical responses of $\mathrm{CdTe} / \mathrm{CdS}$ core/shell quantum dots. Synth Reactivity Inorg Metal-Organic, Nano-Metal Chem (2012) 42(8):1060-3. doi:10.1080/ 15533174.2011 .614996

65. Zielinski M, Oron D, Chauvat D, and Zyss J. Second-harmonic generation from a single core/shell quantum dot. Small (2009) 5(24):2835-40. doi:10. 1002/smll.200900399

66. Brus L. Electronic wave functions in semiconductor clusters: experiment and theory. J Phys Chem (1986) 90(12):2555-60. doi:10.1021/j100403a003 
67. Nandan Y, and Mehata MS. Wavefunction engineering of type-I/Type-II excitons of CdSe/CdS core-shell quantum dots. Sci Rep (2019) 9(1). doi:10. 1038/s41598-018-37676-3

68. Ratnesh RK, and Mehata MS. Controlled synthesis and optical properties of tunable CdSe quantum dots and effect of pH. AIP Adv (2015) 5(9):097114. doi:10.1063/1.4930586

69. Kazes M, Lewis DY, Ebenstein Y, Mokari T, and Banin U. Lasing from semiconductor quantum rods in a cylindrical microcavity. Adv Mater () 14(4):317-21. doi:10.1002/1521-4095(20020219)14:4<317::AIDADMA317>3.0.CO;2-U

70. Wang T, Zhu B, Wang S, Yuan Q, Zhang H, and Kang Z. Influence of shell thickness on the performance of NiO-based all-inorganic quantum dot lightemitting diodes. ACS Appl Mater Inter (2018) 10(17):14894-900. doi:10. 1021/acsami.8b01814

71. Ratnesh RK, and Mehata MS. Synthesis and optical properties of core-multishell CdSe/CdS/ZnS quantum dots: surface modifications. Opt Mater (2017) 64:250-6. doi:10.1016/j.optmat.2016.11.043

72. Ratnesh RK, and Mehata MS. Investigation of biocompatible and protein sensitive highly luminescent quantum dots/nanocrystals of CdSe, CdSe/ZnS and CdSe/CdS. Spectrochim Acta A Mol Biomol Spectrosc (2017) 179:201-10. doi:10.1016/j.saa.2017.02.028

73. Bruchez M, Moronne M, Gin P, Weiss S, and Alivisatos AP. Semiconductor nanocrystals as fluorescent biological labels. Science (1998) 281(5385): 2013-6. doi:10.1126/science.281.5385.2013

74. Chan WC, and Nie S. Quantum dot bioconjugates for ultrasensitive nonisotopic detection. Science (1998) 281(5385):2016-8. doi:10.1126/ science.281.5385.2016

75. Mehata MS. Enhancement of charge transfer and quenching of photoluminescence of capped CdS quantum dots. Sci Rep (2015) 5(1): 1-11. doi:10.1038/srep12056

76. De Geyter B, Justo Y, Moreels I, Lambert K, Smet PF, and Van Thourhout D. The different nature of band edge absorption and emission in colloidal PbSe/ CdSe core/shell quantum dots. ACS Nano (2011) 5(1):58-66. doi:10.1021/ nn102980e

77. AbouElhamd A, Al-Sallal K, and Hassan A. Review of core/shell quantum dots technology integrated into building's glazing. Energies (2019) 12:1058-6. doi:10.3390/en12061058

78. Ebrahim Jasim K. Third-order nonlinear optical properties of quantum dots. In: Standards, methods and solutions of metrology. London, United Kingdom: IntechOpen (2019).

79. Jasim KE. Quantum dots solar cells. In: Solar cells - new approaches and reviews. London, United Kingdom: InTech (2015).

80. Kayanuma Y. Quantum-size effects of interacting electrons and holes in semiconductor microcrystals with spherical shape. Phys Rev B Condens Matter (1988) 38(14):9797-805. doi:10.1103/PhysRevB.38.9797

81. Roussignol P, Ricard D, and Flytzanis C. Quantum confinement mediated enhancement of optical kerr effect in CdS x Sel?x semiconductor microcrystallites. Appl Phys B (1990) 51(6):437-42. doi:10.1007/BF00329108

82. Thomas D, Lee HO, Santiago KC, Pelzer M, Kuti A, and Jenrette E. Rapid microwave synthesis of tunable cadmium selenide (CdSe) quantum dots for optoelectronic applications. J Nanomater (2020) 2020:1. doi:10.1155/2020/ 5056875

83. Klimov VI, Mikhailovsky AA, Xu S, Malko A, Hollingsworth JA, and Leatherdale CA. Optical gain and stimulated emission in nanocrystal quantum dots. Science () 290(5490):314-7. doi:10.1126/science.290.5490.314

84. Harel E, Rupich SM, Schaller RD, Talapin DV, and Engel GS. Measurement of electronic splitting in $\mathrm{PbS}$ quantum dots by two-dimensional nonlinear spectroscopy. Phys Rev B (2012) 86(7):075412. doi:10.1103/PhysRevB.86.075412

85. Vasudevan D, Gaddam RR, Trinchi A, and Cole I. Core-shell quantum dots: properties and applications. J Alloys Compounds (2015) 636:395-404. doi:10. 1016/j.jallcom.2015.02.102

86. Nideep TK, Ramya M, Nampoori VPN, and Kailasnath M. Optical limiting and optical properties of water soluble CdTe quantum dots prepared through a colloidal chemical route. Optik (Stuttg). (20182019) 179:1101-8. doi:10. 1016/j.ijleo.2018.10.168

87. Park SH, Morgan RA, Hu YZ, Lindberg M, Koch SW, and Peyghambarian N. Nonlinear optical properties of quantum-confined CdSe microcrystallites. J Opt Soc Am B (19901990) 7(10):2097. doi:10.1364/josab.7.002097
88. Mathew S, Saran AD, Singh Bhardwaj B, Ani Joseph S, Radhakrishnan P, and Nampoori VPN. Size dependent optical properties of the CdSe-CdS coreshell quantum dots in the strong confinement regime. J Appl Phys (2012) 111(7):074312. doi:10.1063/1.3702430

89. Kamat PV, and Dimitrijevic NM. Photoelectrochemistry in semiconductor particulate systems. 13. Surface modification of cadmium sulfide semiconductor colloids with diethyldithiocarbamate. J Phys Chem (1989) 93(10):4259-63. doi:10.1021/j100347a069

90. Kamat PV, de Lind van Wijngaarden M, and Hotchandani S. Surface modification of CdS colloids with mercaptoethylamine. Isr J Chem (1993) 33(1):47-51. doi:10.1002/ijch.199300008

91. Kamat PV, Dimitrijevic NM, and Fessenden RW. Photoelectrochemistry in particulate systems. 6. Electron-transfer reactions of small cadmium sulfide colloids in acetonitrile. J Phys Chem (1987) 91(2):396-401. doi:10.1021/ j100286a029

92. Vossmeyer T, Katsikas L, Giersig M, Popovic IG, Diesner K, and Chemseddine A. CdS nanoclusters: synthesis, characterization, size dependent oscillator strength, temperature shift of the excitonic transition energy, and reversible absorbance shift. J Phys Chem (1994) 98(31):7665-73. doi:10.1021/j100082a044

93. Henari FZ, Morgenstern K, Blau WJ, Karavanskii VA, and Dneprovskii VS. Third-order optical nonlinearity and all-optical switching in porous silicon. Appl Phys Lett (1995) 67:323. doi:10.1063/1.115432

94. Yu B, Zhu C, and Gan F. Optical nonlinearity of $\mathrm{Bi} 2 \mathrm{O} 3$ nanoparticles studied by Z-scan technique. J Appl Phys (1997) 82(9):4532-7. doi:10.1063/1.366189

95. Wang Y. Nonlinear optical properties of nanometer-sized semiconductor clusters. Acc Chem Res (1991) 24(5):133-9. doi:10.1021/ar00005a002

96. Hora H, and Shen YR. The principles of nonlinear optics. New York: John Wiley \& Sons (1984). p. 576. doi:10.1017/s0263034600001889

97. Jacobsohn M, and Banin U. Size dependence of second harmonic generation in CdSe nanocrystal quantum dots. J Phys Chem B (2000) 104(1):1-5. doi:10. 1021/jp9925076

98. Cirloganu CM, Cox N, Ensley T, Hu H, Nootz G, Olszak PD, et al. ThreePhoton absorption spectra and bandgap scaling in direct-gap semiconductors. arXiv (2020) 7(8):888-99. doi:10.1364/optica.396056

99. Takagahara T. (1991). Dependence on dimensionality of excitonic optical nonlinearity in quantum confined structures. Solid State Commun., 78(4), 279-282. doi:10.1016/0038-1098(91)90197-4 (Accessed Aug. 22, 2020).

100. Takagahara T. Quantum dot lattice and enhanced excitonic optical nonlinearity. Surf Sci (1992) 267(1-3):310-4. doi:10.1016/0039-6028(92) 91144-Z

101. Kayanuma Y. Resonant interaction of photons with a random array of quantum dots. J Phys Soc Jpn (1993) 62(1):346-56. doi:10.1143/JPSJ. 62.346

102. Poles E, Selmarten DC, Mićić OI, and Nozik AJ. Anti-Stokes photoluminescence in colloidal semiconductor quantum dots. Appl Phys Lett (1999) 75(7):971-3. doi:10.1063/1.124570

103. Anand B, Krishnan SR, Podila R, Sai SS, Rao AM, and Philip R. The role of defects in the nonlinear optical absorption behavior of carbon and $\mathrm{ZnO}$ nanostructures. Phys Chem Chem Phys (2014) 16(18):8168-77. doi:10.1039/ c3cp55334e

104. Alivisatos AP, Harris TD, Carroll PJ, Steigerwald ML, and Brus LE. Electron-vibration coupling in semiconductor clusters studied by resonance Raman spectroscopy. J Chem Phys (1989) 90(7):3463-8. doi:10.1063/1.455855

105. Cao Y, Wang C, Zhu B, and Gu Y. A facile method to synthesis high-quality CdSe quantum dots for large and tunable nonlinear absorption. Opt Mater (2017) 66:59-64. doi:10.1016/j.optmat.2017.01.041

106. Majles Ara MH, Moslemi Z, Naderi H, Mihandoost A, Daneshfar A, and Sahraei R. Nonlinear optical properties of CdTe nanocrystals synthesized by a green room temperature solution method. Appl Phys B (2015) 118(4):567-72. doi:10.1007/s00340-015-6040-1

107. Garmire E. Nonlinear optics in daily life. Opt Express (2013) 21(25):30532. doi:10.1364/oe.21.030532

108. Feng X, Xiong G, Zhang $\mathrm{X}$, and Gao H. Third-order nonlinear optical susceptibilities associated with intersubband transitions in CdSe/ZnS coreshell quantum dots. Physica B: Condensed Matter (2006) 383(2):207-12. doi:10.1016/j.physb.2006.03.012 
109. Cox JD, Singh MR, von Bilderling C, and Bragas AV. A nonlinear switching mechanism in quantum dot and metallic nanoparticle hybrid systems. Adv Opt Mater (2013) 1(6):460-7. doi:10.1002/adom.201300105

110. Britt J, and Ferekides C. Thin-film CdS/CdTe solar cell with $15.8 \%$ efficiency. Appl Phys Lett (1993) 62(22):2851-2. doi:10.1063/1.109629

111. Xiong S, Xi B, and Qian Y. CdS hierarchical nanostructures with tunable morphologies: preparation and photocatalytic properties. J Phys Chem C (2010) 114(33):14029-35. doi:10.1021/jp1049588

112. Duan X, Niu C, Sahi V, Chen J, Parce JW, and Empedocles S. Highperformance thin-film transistors using semiconductor nanowires and nanoribbons. Nature (2003) 425(6955):274-8. doi:10.1038/nature01996

113. Pardo-Yissar V, Katz E, Wasserman J, and Willner I. Acetylcholine esteraselabeled CdS nanoparticles on electrodes: photoelectrochemical sensing of the enzyme inhibitors. J Am Chem Soc (2003) 125(3):622-3. doi:10.1021/ ja028922k

114. Pan H, Chu H, Li Y, Qi N, Zhao S, and Li G. Nonlinear optical properties of colloidal CdSe/ZnS quantum dots in PMMA. Nanotechnology (2020) 31: 195703-19. doi:10.1088/1361-6528/ab7045

115. Oldenburg SJ, Averitt RD, Westcott SL, and Halas NJ. Nanoengineering of optical resonances (1998).[Online]. Available: https://www.sciencedirect. com/science/article/pii/S0009261498002772. (Accessed Jul. 12, 2020).

116. Kalele S, Gosavi SW, Urban J, and Kulkarni SK. Nanoshell particles: synthesis, properties and applications (2006).

117. Rosencher E, and Bois P. Model system for optical nonlinearities: asymmetric quantum wells. Phys Rev B Condens Matter (1991) 44(20):11315-27. doi:10. 1103/PhysRevB.44.11315

118. Sundar VC, Eisler H-J, Bawendi MG, and Room-Temperature “. Roomtemperature, tunable gain media from novel II-VI nanocrystal-titania composite matrices. Adv Mater (2002) 14(10):739-43. doi:10.1002/15214095(20020517)14:10<739::aid-adma739>3.0.co;2-y

119. Kortan AR, Hull R, Opila RL, Bawendi MG, Steigerwald ML, and Carroll PJ. Nucleation and growth of CdSe on ZnS quantum crystallite seeds, and vice versa, in inverse micelle media. J Am Chem Soc (1990) 112(4):1327-32. doi:10.1021/ja00160a005

120. Mews A, Eychmueller A, Giersig M, Schooss D, and Weller H. Preparation, characterization, and photophysics of the quantum dot quantum well system cadmium sulfide/mercury sulfide/cadmium sulfide. J Phys Chem (1994) 98(3):934-41. doi:10.1021/j100054a032

121. Lu Y, Yin Y, Li Z-Y, and Xia Y. Synthesis and self-assembly of Au@ SiO2Core-Shell colloids. Nano Lett (2002) 2(7):785-8. doi:10.1021/ nl025598i

122. Lu W, Wang B, Zeng J, Wang X, Zhang S, and Hou JG. Synthesis of core/shell nanoparticles of $\mathrm{Au} / \mathrm{CdSe}$ via Au-Cd bialloy precursor. Langmuir (2005) 21(8):3684-7. doi:10.1021/la0469250

123. Çadirci M, Gündoğdu Y, Elibol E, and Kılıç HŞ. Nonlinear optical properties of core shell type II quantum dot structures. Opt Laser Tech (2020) 128(March):106246. doi:10.1016/j.optlastec.2020.106246

124. Yu WW, Qu L, Guo W, and Peng X. Experimental determination of the extinction coefficient of CdTe, CdSe, and CdS nanocrystals. Chem Mater (2003) 15(14):2854-60. doi:10.1021/cm034081k

125. Tyrrell EJ, and Smith JM. Effective mass modeling of excitons in type-II quantum dot heterostructures. Phys Rev B (2011) 84(16):165328. doi:10.1103/ PhysRevB.84.165328

126. Geethakrishnan T, and Palanisamy PK. Z-scan determination of the thirdorder optical nonlinearity of a triphenylmethane dye using $633 \mathrm{~nm} \mathrm{He}-\mathrm{Ne}$ laser. Opt Commun (2007) 270(2):424-8. Feb. 2007. doi:10.1016/j.optcom. 2006.09.035

127. Singh V, Aghamkar P, and Z-scan ". Z-scan: a simple technique for determination of third-order optical nonlinearity. AIP Conf Proc (2015) 1675. doi:10.1063/1.4929311

128. Bhagyaraj S, Perumbilavil S, Udayabashkar R, Mangalaraja RV, Thomas S, and Kalarikkal N. Tuning of nonlinear absorption in highly luminescent CdSe based quantum dots with core-shell and core/multi-shell architectures. Phys Chem Chem Phys (2019) 21(21):11424-34. doi:10.1039/c9cp00476a

129. Perumbilavil S, López-Ortega A, Tiwari GK, Nogués J, Endo T, and Philip R. Enhanced ultrafast nonlinear optical response in ferrite core/shell nanostructures with excellent optical limiting performance. Small (2018) 14(6). doi:10.1002/smll.201701001
130. Pu SC, Yang MJ, Hsu CC, Lai CW, Hsieh CC, and Lin SH. The empirical correlation between size and two-photon absorption cross section of CdSe and CdTe quantum dots. Small (2006) 2(11):1308-13. doi:10.1002/smll. 200600157

131. Liu Y, Chen P, Wang Z-H, Bian F, Lin L, and Chang S-J. Efficient two-photon absorption of CdSe-CdS/ZnS core-multishell quantum dots under the excitation of near-infrared femtosecond pulsed laser. Laser Phys (2009) 19(9):1886-90. doi:10.1134/s1054660x09170113

132. Banfi GP, Degiorgio V, Fortusini D, and Bellini M. Measurement of the twophoton absorption coefficient of semiconductor nanocrystals by using tunable femtosecond pulses. Opt Lett (1996) 21(18):1490-2. doi:10.1364/ ol.21.001490

133. Nathan V, Mitra SS, and Guenther AH. Review of multiphoton absorption in crystalline solids. J Opt Soc Am B (1985) 2(2):294. doi:10.1364/josab.2.000294

134. Dakovski GL, and Shan J. Size dependence of two-photon absorption in semiconductor quantum dots. J Appl Phys (2013) 114(1):014301014301. doi:10.1063/1.4811349

135. Valligatla S, Haldar KK, Patra A, and Desai NR. Nonlinear optical switching and optical limiting in colloidal CdSe quantum dots investigated by nanosecond Z-scan measurement. Opt Laser Tech (2016) 84:87-93. doi:10. 1016/j.optlastec.2016.05.009

136. Sreeramulu V, Haldar KK, Patra A, and Rao DN. Nonlinear optical switching and enhanced nonlinear optical response of Au-CdSe heteronanostructures. J Phys Chem C (2014) 118(51):30333-41. doi:10.1021/jp5118739

137. Venkatram N, Sathyavathi R, and Rao DN. Size dependent multiphoton absorption and refraction of CdSe nanoparticles. Opt Express (2007) 15(19): 12258. doi:10.1364/oe.15.012258

138. Chang J. H., Hahm D., Char K., and Bae W. K., "Interfacial engineering of core/shell heterostructured nanocrystal quantum dots for light-emitting applications," Journal of Information Display, vol. 18, no. 2, pp. 57-65. 2017, doi:10.1080/15980316.2017.1313179

139. Supran GJ, Song KW, Hwang GW, Correa RE, Scherer J, and Dauler EA. High-performance shortwave-infrared light-emitting devices using core-shell (PbS-CdS) colloidal quantum dots. Adv Mater Weinheim (2015) 27(8): 1437-42. doi:10.1002/adma.201404636

140. Wang Y, Ding G, Mao JY, Zhou Y, and Han ST. Recent advances in synthesis and application of perovskite quantum dot based composites for photonics, electronics and sensors. Sci Tech Adv Mater (2020) 21(1):278-302. doi:10. 1080/14686996.2020.1752115

141. Mashford BS, Stevenson M, Popovic Z, Hamilton C, Zhou Z, and Breen C. High-efficiency quantum-dot light-emitting devices with enhanced charge injection. Nat Photon (2013) 7(5):407-12. doi:10.1038/nphoton.2013.70

142. Órdenes-Aenishanslins N, Anziani-Ostuni G, Quezada CP, EspinozaGonzález R, Bravo D, and Pérez-Donoso JM. Biological synthesis of CdS/ CdSe core/shell nanoparticles and its application in quantum dot sensitized solar cells. Front Microbiol (2019) 10(JULY). doi:10.3389/fmicb.2019.01587

143. Feng H-P. Historical perspective Core-shell nanomaterials: applications in energy storage and conversion. Elsevier (2019). doi:10.1016/j.cis.2019.03.001

144. Kostopoulou A, Brintakis K, Nasikas NK, and Stratakis E. Perovskite nanocrystals for energy conversion and storage. Nanophotonics (0120) 8(10):1607-40. doi:10.1515/nanoph-2019-0119

145. Selopal GS, Zhao H, Wang ZM, and Rosei F. Core/shell quantum dots solar cells. Adv Funct Mater (2020) 30(13):1908762. doi:10.1002/adfm.201908762

146. Shu Y, Lin X, Qin H, Hu Z, Jin Y, and Peng X. Quantum dots for display applications. In: Angewandte chemie - international edition, Vol. 59. Wiley-VCH Verlag (2020). p. 22312-23. doi:10.1002/anie.202004857

147. Li Y, Zhao Y, Cheng H, Hu Y, Shi G, and Dai L. Nitrogen-doped graphene quantum dots with oxygen-rich functional groups. J Am Chem Soc (2012) 134(1):15-8. doi:10.1021/ja206030c

148. Aldeek F, Mustin C, Balan L, Medjahdi G, Roques-Carmes T, and Arnoux P. Enhanced photostability from $\mathrm{CdSe}(\mathrm{S}) / \mathrm{ZnO}$ core/shell quantum dots and their use in biolabeling. Eur J Inorg Chem (2011) 2011:794-801. doi:10.1002/ ejic.201000790, no

149. Munasinghe E, Aththapaththu M, and Jayarathne L. Magnetic and quantum dot nanoparticles for drug delivery and diagnostic systems. In: Colloid science in pharmaceutical nanotechnology. IntechOpen (2020).

150. Law WC, Yong KT, Roy I, Ding H, Hu R, and Zhao W. Aqueous-phase synthesis of highly luminescent $\mathrm{CdTe} / \mathrm{ZnTe}$ core/shell quantum dots 
optimized for targeted bioimaging. Weinheim, Germany: Small (2009) 5(11): 1302-10. doi:10.1002/smll.200801555

151. A. S.-C. nanotechnology and undefined. In: Nanobio applications of quantum dots in cancer: imaging, sensing, and targeting. Springer (2011). doi:10.1007/ s12645-011-0015-7

152. Nandi S, Malishev R, Parambath Kootery K, Mirsky Y, Kolusheva S, and Jelinek R. Membrane analysis with amphiphilic carbon dots. Chem Commun (Camb) (2014) 50(71):10299-302. doi:10.1039/c4cc03504f

153. Mattoussi $\mathrm{H}$, Palui G, and Na H. Luminescent quantum dots as platforms for probing in vitro and in vivo biological processes. Adv Drug Deliv Rev (2012) 64(2):138-66. doi:10.1016/j.addr.2011.09.011

154. Chen F, and Gerion D. Fluorescent CdSe/ZnS Nanocrystal-Peptide conjugates for long-term, nontoxic imaging and nuclear targeting in living cells. Nano Lett (2004) 4(10):1827-32. doi:10.1021/nl049170q

155. Kalytchuk S, Wang Y, Poláková K, and Zbořil R. Carbon dot fluorescencelifetime-encoded anti-counterfeiting. ACS Appl Mater Inter (2018) 10(35): 29902-8. doi:10.1021/acsami.8b11663

156. Liu Y., Han F., Li F., Zhao Y., Chen M., Xu Z., Zheng X., Hu H., Yao J., Guo T., Lin W., Zheng Y., You B., Liu P., Li Y., and Qian L., "Inkjet-printed unclonable quantum dot fluorescent anti-counterfeiting labels with artificial intelligence authentication," Nat. Commun., vol. 10, no. 1.2019. doi:10.1038/s41467-019-10406-7

157. Zhang F. Synergetic effect of the surfactant and silica coating on the enhanced emission and stability of perovskite quantum dots for anticounterfeiting. ACS Appl Mater Inter (2019) 11(31):28013-22. Accessed: Jan. 16, 2021. [Online]. Available:. https://pubs.acs.org/doi/abs/10.1021/acsami.9b07518

158. Shim J, Ko Y, Lee KS, Partha K, Lee C-H, Yu K, et al. Conductive Co3O4/ graphene (core/shell) quantum dots as electrode materials for electrochemical pseudocapacitor applications. Composites B: Eng (2017) 130:230-5. doi:10. 1016/j.compositesb.2017.07.039 (Accessed Jan. 16, 2021).

159. Chao D, Zhu C, Xia X, Liu J, Zhang X, and Wang J. Graphene quantum dots coated VO2Arrays for highly durable electrodes for $\mathrm{Li}$ and $\mathrm{Na}$ ion batteries. Nano Lett (2015) 15(1):565-73. doi:10.1021/nl504038s

160. Fan H, Zhang M, Bhandari B, and hui Yang C. Food waste as a carbon source in carbon quantum dots technology and their applications in food safety detection. Trends Food Sci Tech (2020) Vol. 95:86-96. doi:10.1016/j.tifs.2019. 11.008

161. Duong H, Rhee JI, and undefined. Use of CdSe/ZnS core-shell quantum dots as energy transfer donors in sensing glucose. Talanta (2007) 73:899, 2007. [Online]. Available:. https://www.sciencedirect.com/science/article/pii/ S0039914007003669. (Accessed Jan. 16, 2021). doi:10.1016/j.talanta. 2007.05.011

162. Gui C, Wang K, Li C, Dai X, and Cui D. A CCD-based reader combined with CdS quantum dot-labeled lateral flow strips for ultrasensitive quantitative detection of CagA. Nanoscale Res Lett (2014) 9(1):1-8. doi:10.1186/1556276X-9-57 (Accessed Jan. 16, 2021).

163. Nugraha MI, Kim H, Sun B, Haque MA, Arquer FPG, and Villalva DR. Lowtemperature-processed colloidal quantum dots as building blocks for thermoelectrics. Adv Energ Mater. (2019) 9(13):1803049. doi:10.1002/ aenm.201803049

164. Peyghambarian N, Fluegel B, Hulin D, Migus A, Joffre M, and Antonetti A. Femtosecond optical nonlinearities of CdSe quantum dots. IEEE J Quan Electron. (1989) 25(12):2516-22. doi:10.1109/3.40636

165. Reiss P, Carayon S, Bleuse J, and Pron A. Low polydispersity core/shell nanocrystals of CdSe/ZnSe and CdSe/ZnSe/ZnS type: preparation and optical studies. Synth Met (2003) 139(3):649-52. doi:10.1016/S0379-6779(03) 00335-7

166. Gerdova I, and Haché A. Third-order non-linear spectroscopy of CdSe and CdSe/ZnS core shell quantum dots. Opt Commun (2005) 246(1-3):205-12. doi:10.1016/j.optcom.2004.10.068

167. Schooss D, Mews A, Eychmüller A, and Weller H. Quantum-dot quantum well CdS/HgS/CdS: theory and experiment. Phys Rev B Condens Matter (1994) 49(24):17072-8. doi:10.1103/PhysRevB.49.17072

168. Augustine AK, Mathew S, Radhakrishnan P, Nampoori VPN, and Kailasnath $M$, "Size dependent optical nonlinearity and optical limiting properties of water soluble CdSe quantum dots," J Nanoscience, 2014, vol. pp. 1-7. 2014, doi:10.1155/2014/623742

169. Nyk M, Szeremeta J, Wawrzynczyk D, and Samoc M. Enhancement of twophoton absorption cross section in CdSe quantum rods. J Phys Chem C (2014) 118(31):17914-21. doi:10.1021/jp501843g

170. Zhang CY, Yeh HC, Kuroki MT, and Wang TH. Single-quantum-dotbased DNA nanosensor. Nat Mater (2005) 4(11):826-31. doi:10.1038/ nmat1508

171. Nozik AJ, Beard MC, Luther JM, Law M, Ellingson RJ, and Johnson JC. Semiconductor quantum dots and quantum dot arrays and applications of multiple exciton generation to third-generation photovoltaic solar cells. Chem Rev (2010) 110(11):6873-90. doi:10.1021/cr900289f

Conflict of Interest: The authors declare that the research was conducted in the absence of any commercial or financial relationships that could be construed as a potential conflict of interest.

Copyright $(2021 \mathrm{Kalsoom}, \mathrm{Yi}, \mathrm{Qu}$ and Liu. This is an open-access article distributed under the terms of the Creative Commons Attribution License (CC BY). The use, distribution or reproduction in other forums is permitted, provided the original author(s) and the copyright owner(s) are credited and that the original publication in this journal is cited, in accordance with accepted academic practice. No use, distribution or reproduction is permitted which does not comply with these terms. 